\title{
Zivilgesellschaftliches Engagement
}

\section{Corinna Kausmann DZA | Luise Burkhardt DIW (SOEP) | Boris Rump DOSB | Nadiya Kelle DZA | Julia Simonson DZA | Clemens Tesch-Römer DZA}

Wer sind die Menschen, die innerhalb und außerhalb zivilgesellschaftlicher Organisationen engagiert sind? Wie hoch ist der Anteil dieser Personen an der Bevölkerung? In welchen gesellschaftlichen Bereichen sind engagierte Menschen tätig? Im vorliegenden Kapitel werden die vorangehenden Darstellungen zivilgesellschaftlicher Organisationen (siehe Kapitel 3) um die Akteure ergänzt, die in diesen Organisationen tätig sind. So sollen zum einen die Engagementquoten im Daten- und Zeitvergleich betrachtet und zum anderen dargestellt werden, in welchen gesellschaftlichen Bereichen sich Menschen engagieren, welche regionalen Unterschiede (Bundesländer) es gibt und wie sich das Engagement zwischen Bevölkerungsgruppen (nach Altersgruppen, Geschlecht, Migrationshintergrund, Bildung und Erwerbsstatus) unterscheidet. Als spezifische Formen des Engagements sollen organisationsgebundenes und informelles Engagement, zeitintensives Engagement sowie Freiwilligendienste betrachtet werden.

Begriffsklärungen und Definitionen: In der öffentlichen Diskussion werden die Begriffe freiwilliges Engagement, bürgerschaftliches Engagement, Ehrenamt und Freiwilligenarbeit häufig synonym verwendet. Obwohl diese Begriffe überlappende Bedeutungen haben, sind doch deutliche Unterschiede erkennbar. Deshalb werden sie im Folgenden beschrieben.

- Bürgerschaftliches Engagement: Als Bezugspunkt dient häufig die Definition der Enquete-Kommission „Zukunft des Bürgerschaftlichen Engagements“, die im Jahr 2002 fünf Kriterien vorlegte: Bürgerschaftliches Engagement ist freiwillig, findet im öffentlichen Raum statt, ist gemeinschaftsbezogen, trägt zum Allgemeinwohl bei und ist nicht auf materiellen Gewinn gerichtet (Deutscher Bundestag 2002). Auch die Sachverständigenkommission des Ersten Engagementberichts der Bundesregierung baute auf diesem Kriterienkatalog auf (Deutscher Bundestag 2012).

(C) Der/die Autor(en) 2019

H. Krimmer (Hrsg.), Datenreport Zivilgesellschaft, Bürgergesellschaft und Demokratie, https://doi.org/10.1007/978-3-658-22958-0_4 
- Ehrenamt: Der Begriff Ehrenamt bezeichnet die Ausübung eines Amtes oder einer Funktion im formalen Rahmen einer zivilgesellschaftlichen Organisation. Ehrenamtliches Engagement ist eine Teilmenge bürgerschaftlichen Engagements: Alle ehrenamtlich Engagierten sind bürgerschaftlich engagiert, aber nicht alle bürgerschaftlich engagierten Personen sind im Rahmen eines Ehrenamts tätig (Stricker 2011; Roth 2000).

- Freiwilligenarbeit: Der Ausdruck Freiwilligenarbeit bezieht sich auf Tätigkeiten, die als Arbeit bezeichnet werden, im Gegensatz zur Erwerbsarbeit aber ohne finanzielle Vergütung erbracht werden (International Labour Organization, ILO 2011, S. 5). Unter dem Begriff der Freiwilligenarbeit werden alle Arten unbezahlter Tätigkeiten zusammengefasst, sowohl im öffentlichen Raum als auch im privaten, familiären Umfeld erbrachte Tätigkeiten (International Labour Organization, ILO 2011). Die Besonderheit dieses Ansatzes ist die Möglichkeit, dem Engagement einen ökonomischen Wert zuzuordnen, um auf diese Weise den Beitrag freiwilliger Arbeit zur Gesamtwirtschaftsleistung einer Gesellschaft darzustellen („establish the economic value of volunteering“, UN General Assembly 2001). Die Konzepte Freiwilligenarbeit und bürgerschaftliches Engagement überlappen zu großen Teilen, aber es gibt Tätigkeiten, die als Freiwilligenarbeit, nicht aber als bürgerschaftliches Engagement (sowie Tätigkeiten, die als bürgerschaftliches Engagement, nicht aber als Freiwilligenarbeit) zu werten sind.

- Freiwilliges Engagement: Das Konzept „Freiwilliges Engagement“ bildet die Grundlage des Freiwilligensurveys. Es orientiert sich ebenfalls an den Kriterien der Enquete-Kommission: Freiwilliges Engagement umfasst Tätigkeiten, die unentgeltlich, freiwillig, im öffentlichen Rahmen und gemeinschaftlich mit anderen Personen ausgeführt werden (Simonson et al. 2017b). Das Kriterium der Gemeinwohlorientierung wird in den Erhebungen des Freiwilligensurveys vorausgesetzt, da in der Befragung und der anschließenden Prüfung der Tätigkeiten eine Einschätzung von Nutzen und auch Kosten der freiwilligen Tätigkeit für das Gemeinwohl nicht möglich ist. Freiwilliges Engagement kann die unterschiedlichsten Formen annehmen.

Der Begriff des zivilgesellschaftlichen Engagements ist ein Oberbegriff für diese vielfältigen Varianten von Engagement und umfasst die oben genannten Engagement-Definitionen (vgl. von Rosenbladt 2001). Einige wenige Beispiele für zivilgesellschaftliches Engagement sind politische Aktivitäten, Aufgaben innerhalb von Kommunen, Interessenvertretungen sowie Arbeiten in Vereinen oder in Elternvertretungen. Mithilfe von Engagementquoten kann diese Fülle an Formen zivilgesellschaftlichen Engagements gebündelt und sichtbar gemacht werden. 
Datengrundlagen zur Erfassung von Engagement: Ebenso wie es verschiedene Formen des freiwilligen Engagements gibt, sind auch die Möglichkeiten vielfältig, die Engagementbeteiligung zu erfragen. Definition und Erfassung von zivilgesellschaftlichem Engagement unterscheiden sich in verschiedenen Studien zum Teil deutlich. Daher können Engagementquoten je nach verwendeter Datengrundlage unterschiedlich ausfallen. Um die Bandbreite verschiedener Messkonzepte und Bereiche von Engagement möglichst gut abzubilden, werden in diesem Kapitel Daten des Deutschen Freiwilligensurveys (FWS), des Deutschen Alterssurveys (DEAS), des Sozio-oekonomischen Panels (SOEP), des Sportentwicklungsberichts (SEB) sowie der Zeitverwendungserhebung (ZVE) dargestellt.

- Deutscher Freiwilligensurvey (FWS): In der telefonischen Erhebung des Freiwilligensurveys wird in einem zweistufigen Vorgehen nach freiwilligem Engagement gefragt. Begonnen wird mit einer Frage nach Aktivitäten, an denen sich eine befragte Person außerhalb von Beruf und Familie in einem (oder mehreren) von 14 gesellschaftlichen Bereichen beteiligt. Wird von der befragten Person eine aktive Beteiligung in mindestens einem der Bereiche angegeben, wird in einer Anschlussfrage ermittelt, ob der oder die Befragte in dem jeweiligen Bereich eine ehrenamtliche Tätigkeit ausübt oder in Vereinen, Initiativen, Projekten oder Selbsthilfegruppen Aufgaben übernimmt. Während es bei der Frage nach den Aktivitäten um das „Mitmachen“ geht, werden bei der Frage nach dem Engagement bestimmte Aufgaben und Arbeiten erfasst (Simonson et al. 2017a). Es wird nachgefragt, ob die Aufgaben oder Arbeiten, die übernommen werden, freiwillig und unbezahlt sind (bzw. nur mit einer geringen Aufwandsentschädigung vergütet werden).

- Deutscher Alterssurvey (DEAS): Im Deutschen Alterssurvey wird ehrenamtliches Engagement in mündlichen Interviews ermittelt. Auch hierbei handelt es sich um eine zweistufige Abfrage, jedoch ausschließlich zu organisationsgebundenem Engagement. Es wird in einem ersten Schritt die Mitgliedschaft in einer Gruppe oder Organisation erfragt. Befragte im Alter von 50 Jahren und älter werden dabei zunächst nach der Mitgliedschaft in einer Gruppe oder Organisation für ältere Menschen gefragt, danach nach einer Mitgliedschaft in einer altersunabhängigen Gruppe oder Organisation. Befragte, die jünger als 50 Jahre sind, erhalten nur die Frage nach altersunabhängigen Gruppen oder Organisationen. In einem zweiten Schritt wird gefragt, ob Mitglieder eine Funktion oder ein Ehrenamt innerhalb der Gruppe, in der sie Mitglied sind, übernommen haben. Als ehrenamtlich engagiert gelten Personen, die sowohl Mitglied sind als auch eine Funktion oder ein Ehrenamt übernommen haben (Wetzel und Simonson 2017). 
- Sozio-oekonomisches Panel (SOEP): In dem Personenfragebogen des Soziooekonomischen Panels wird Ehrenamt in Verbindung mit dem Thema Freizeit erhoben. Dabei wird gefragt, ob man ehrenamtliche Tätigkeiten in Vereinen, Verbänden oder sozialen Diensten täglich, mindestens einmal pro Woche, mindestens einmal pro Monat, seltener oder nie ausübt (TNS Infratest Sozialforschung 2014).

- Sportentwicklungsbericht (SEB): Der Sportentwicklungsbericht ist von seiner Anlage und Methodik eine organisationsgebundene Untersuchung von eingetragenen Sportvereinen (e. V.), in der die befragten Vereine mittels einer Online-Befragung unter anderem Auskunft über das Engagement der ehrenamtlich Aktiven und freiwilligen HelferInnen aus ihrem Sportverein geben. Damit steht nicht das Engagement der befragten Einzelperson(en) im Mittelpunkt der Untersuchung, sondern die Fragen beziehen sich vielmehr auf den Sportverein selbst und seine Mitglieder insgesamt. In der sechsten Welle des SEB 2015/2016 wurden im Themenbereich freiwilliges Engagement und Ehrenamt Fragen zu den unterschiedlichen ehrenamtlichen Positionen (FunktionsträgerInnen, TrainerInnen, ÜbungsleiterInnen, SchiedsrichterInnen etc.) und deren Entwicklung, über den geleisteten Stundenumfang pro Monat sowie über die durchgeführten Maßnahmen zur Bindung und Gewinnung der ehrenamtlich Engagierten im Sportverein gestellt. Ab der siebten Welle (Sportentwicklungsbericht 3.0) wird die Vereinsbefragung durch ausgewählte Stakeholderanalysen (zum Beispiel über die Zielgruppe der TrainerInnen) erweitert.

- Zeitverwendungserhebung (ZVE): Im Rahmen der Zeitverwendungserhebung wird Engagement mittels eines Personenfragebogens und auf Basis von Tagebuchaufzeichnungen berücksichtigt. In dem Tagebuch wird die Zeit für „Ehrenamt, freiwilliges Engagement“ protokolliert. Der Fragebogen enthält zwei Fragen zum Engagement: „Waren Sie in den letzten zwölf Monaten in folgenden Bereichen ehrenamtlich oder freiwillig tätig? Die ehrenamtlichen oder freiwilligen Tätigkeiten umfassen nur unbezahlte oder gegen eine geringe Aufwandsentschädigung ausgeübte Tätigkeiten“ sowie "Wie viel Zeit wenden Sie für Ihr gesamtes ehrenamtliches oder freiwilliges Engagement im Durchschnitt pro Monat auf?" (Burkhardt et al. 2017; Statistisches Bundesamt 2015). 


\subsection{Engagementquote}

Im Folgenden wird die Engagementbeteiligung in Deutschland seit 1999 zunächst anhand der Daten des Deutschen Freiwilligensurveys (FWS) dargestellt. Anschließend werden mithilfe des FWS, des Deutschen Alterssurveys (DEAS) und des Sozio-oekonomischen Panels (SOEP) exemplarisch die spezifischen Zugänge dieser Studien und die daraus resultierenden Engagementquoten betrachtet.

Engagementquote im Deutschen Freiwilligensurvey (FWS): Die Beteiligungsquote im Engagement der Wohnbevölkerung Deutschlands im Alter von 14 und mehr Jahren beträgt im Deutschen Freiwilligensurvey 2014 insgesamt 43,6 Prozent. In der ersten Befragung im Jahr 1999 lag die Engagementquote noch bei 34,o Prozent (Abb. 4.1). In Abbildung 4.1 sind jeweils zwei Engagementquoten für jedes Erhebungsjahr angegeben. Die jeweils höheren Werte - Quoten vor Prüfung - beziehen sich auf die „ungeprüften“ Engagementangaben (hier werden alle Angaben der Befragten gezählt, ohne zu prüfen, ob diese den Kriterien des freiwilligen Engagements entsprechen). Die jeweils niedrigen Werte in Abbildung 4.1 - Quoten nach Prüfung - beziehen sich auf die "geprüften“ Engagementangaben (hier werden nur jene Angaben der Befragten gezählt, die den Kriterien des freiwilligen Engagements entsprechen; Simonson et al. 2017a). Die Beteiligung im freiwilligen Engagement ist, der Quote vor Prüfung zufolge, von 1999 bis 2014 kontinuierlich angestiegen (1999: 35,3 Prozent, 2004: 37,6 Prozent, 2009: 39,8 Prozent, 2014: 44,3 Prozent). Die Quote nach Prüfung, die Anteile der Personen, die auch nach inhaltlicher Prüfung als „engagiert“ bewertet wurden, zeigt ein anderes Bild. Zwischen 1999 über 2004 bis 2009 ist dabei ein moderater Anstieg zu verzeichnen. Von 2009 zu 2014 fällt der Anstieg im Vergleich dazu stärker aus. Während sich die Beteiligung im freiwilligen Engagement von 1999 bis 2014 innerhalb von 15 Jahren um 9,6 Prozentpunkte erhöht hat, ist die Beteiligung von 2009 bis 2014 innerhalb von fünf Jahren um 7,7 Prozentpunkte angestiegen. Vermutlich ist das methodische Vorgehen bei der Engagementprüfung im Jahr 2009 der Grund für die unterschiedlichen Verläufe der Quoten vor und nach Prüfung.

Die steigenden Anteile freiwilligen Engagements in der Wohnbevölkerung Deutschlands lassen sich mit verschiedenen gesellschaftlichen Entwicklungen erklären. Die Bildungsexpansion, die gestiegene Erwerbsbeteiligung, insbesondere von Frauen, sowie die wachsende Anzahl von Vereinen sind die wichtigsten dieser Entwicklungen, die zu mehr Gelegenheiten und Anknüpfungspunkten zum freiwilligen Engagement und damit auch zu einer Dynamisierung des Anstiegs

1 Dieses Unterkapitel wurde verfasst von Corinna Kausmann, DZA; Nadiya Kelle, DZA; Julia Simonson, DZA und Clemens Tesch-Römer, DZA. 
Abbildung 4.1 Anteile freiwillig engagierter Personen im Zeitvergleich (in Prozent)

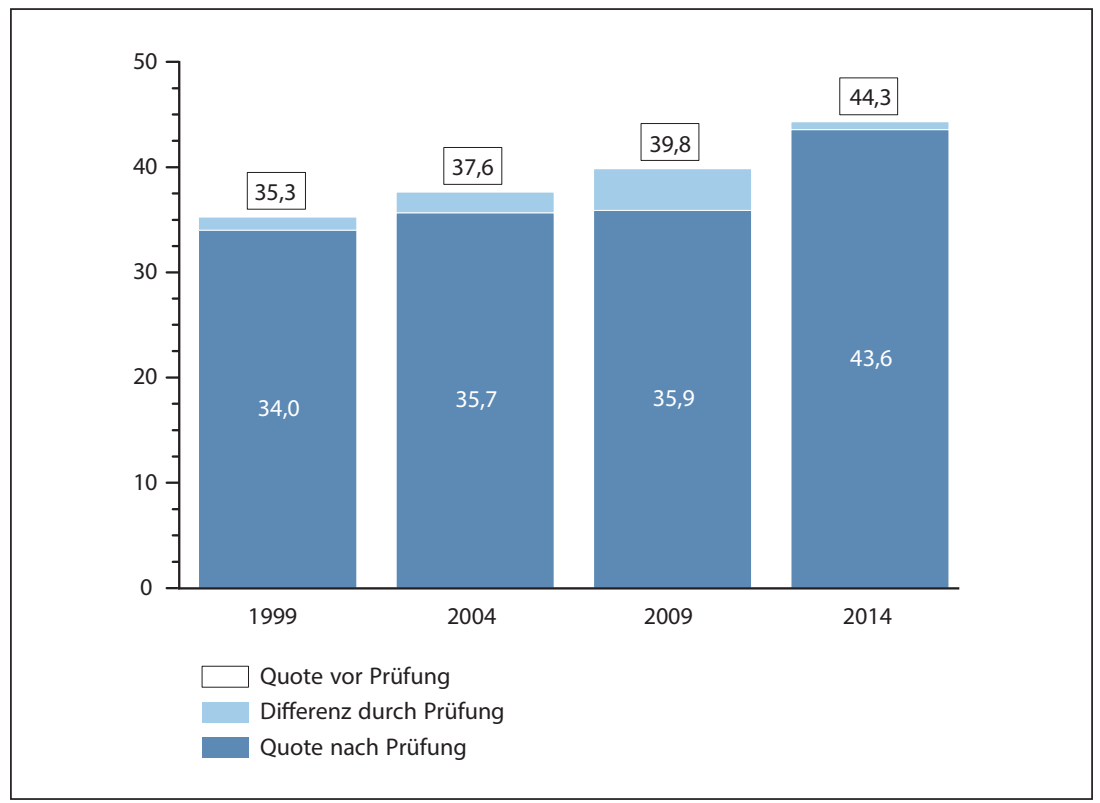

Quelle: FWS, gewichtet, Berechnungen DZA. Basis: Alle Befragten. FWS 1999 ( $n=14922$ ), FWS 2004 $(n=15000)$, FWS 2009 ( $n=20005)$, FWS $2014(n=28689)$.

Nachrichtlich: Bei der Quote vor Prüfung im Jahr 1999 handelt es sich um eine Schätzung.

von 2009 zu 2014 geführt haben können (Vogel et al. 2017a). Auch der demografische Wandel bringt Veränderungen bezüglich des Engagements mit sich. So gibt es auf der einen Seite in Deutschland immer weniger junge Menschen, weshalb zivilgesellschaftliche Akteure mehr für sich werben (müssen), um „EngagementNachwuchs" zu erhalten. Dies führt jedoch zu einer erhöhten Aufmerksamkeit und so auch zu mehr Engagierten. Auf der anderen Seite gibt es durch die gestiegene Lebenserwartung mehr ältere Menschen, die aufgrund einer besseren Gesundheit auch im Ruhestand noch häufiger engagiert sind. Zugleich tragen verschiedene politische und zivilgesellschaftliche Akteure durch den Ausbau von Infrastruktur und Rahmenbedingungen für freiwilliges Engagement zum Anstieg der Beteiligung bei. 
Engagementquote in weiteren Datensätzen (DEAS und SOEP): Während der Deutsche Freiwilligensurvey (FWS) im Jahr 2014 eine Engagementquote von 43,6 Prozent ausweist, beträgt laut sozio-oekonomischem Panel (SOEP) 2013 die Engagementquote rund 30 Prozent (Alscher und Priller 2016) und laut Deutschem Alterssurvey (DEAS) 2014 zeigt sich eine Quote von 22,2 Prozent (Wetzel und Simonson 2017). Die Differenzen in den Engagementquoten zwischen dem DEAS, dem SOEP und dem FWS sind zurückzuführen auf: die unterschiedlichen zugrunde liegenden Engagementdefinitionen, unterschiedlich erfasste Zeitrahmen des Engagements und unterschiedliche Grundgesamtheiten der Personen, die für die jeweiligen Studien befragt werden.

- Unterschiedliche Quoten aufgrund unterschiedlicher Engagementdefinition: Die im Vergleich zu DEAS und SOEP höhere Engagementbeteiligung im FWS lässt sich vor allem auf die weite Definition des Engagementbegriffs im FWS zurückführen. Im SOEP werden nur „ehrenamtliche Tätigkeiten in Vereinen, Verbänden oder sozialen Diensten" erfragt (TNS Infratest Sozialforschung 2014). Im DEAS wird nach einer "Funktion oder einem Ehrenamt“ in einer Gruppe oder Organisation gefragt, in der die Person auch Mitglied ist (Wetzel und Simonson 2017). Nach dieser Begrifflichkeit von Engagement sind Tätigkeiten nicht erfasst, die individuell organisiert sind und nicht in einem formal organisierten Rahmen stattfinden. Im DEAS schränkt das Kriterium der Mitgliedschaft den Personenkreis der Engagierten ein, da es auch möglich ist, sich in formalen Organisationen zu engagieren, ohne Mitglied dieser Organisation zu sein. Im FWS kann freiwilliges Engagement dagegen sowohl im formellen als auch in einem informellen Rahmen stattfinden.

- Unterschiedliche Quoten aufgrund unterschiedlicher Zeitrahmen: Auch die unterschiedlichen Zeitrahmen, die in den verschiedenen Surveys vorgegeben werden, schlagen sich wahrscheinlich in den Quoten nieder. Im FWS 2014 ist ein Zeitfenster von zwölf Monaten vorgegeben, um saisonale Schwankungen im Engagement zu berücksichtigen. In der Befragung des SOEP wird nach der Häufigkeit gefragt, mit der die Tätigkeit ausgeübt wird, ohne dass ein Zeitrahmen vorgegeben wird (täglich, mindestens einmal pro Woche, mindestens einmal pro Monat, seltener oder nie). Auch im DEAS wird kein Zeitfenster angegeben. Zum Einstieg wird nach der aktuellen Mitgliedschaft gefragt und daran anschließend „Üben Sie dort eine Funktion aus oder haben Sie ein Ehrenamt inne?" (Engstler et al. 2015). Somit liegt es im Ermessen der Befragten, ob nur aktuelle ehrenamtliche Tätigkeiten genannt werden oder auch Funktionen und Ämter im Rahmen dieser Mitgliedschaft, die zum Beispiel (nur) einmal vor einigen Monaten ausgeübt wurden. 
- Unterschiedliche Quoten aufgrund unterschiedlicher Grundgesamtheiten: Als letzter Aspekt ist auf die unterschiedlichen Grundgesamtheiten in den drei Studien zu verweisen. Im FWS ist die Grundgesamtheit die Wohnbevölkerung Deutschlands ab 14 Jahren, im SOEP die Wohnbevölkerung Deutschlands ab 17 Jahren und im DEAS die Wohnbevölkerung im Alter von 40 bis 85 Jahren. Nur im FWS wird auch die Altersgruppe der 14- bis 16-Jährigen befragt (diese wird weder im SOEP noch im DEAS befragt), deren Beteiligung im Engagement besonders hoch ist (siehe auch Abschnitt 4.4). Möglicherweise schlägt sich also auch die Wahl unterschiedlicher Grundgesamtheiten in den unterschiedlichen Engagementquoten nieder.

Der Vergleich dieser drei Studien und ihrer Ansätze, Engagement zu quantifizieren, zeigt exemplarisch auf, wie sich die unterschiedlichen Konzepte, die auch in der Einleitung dargestellt wurden, auf die Darstellung des (freiwilligen) Engagements in Deutschland auswirken können.

\subsection{Engagement in verschiedenen gesellschaftlichen Bereichen ${ }^{2}$}

Freiwilliges Engagement wird in verschiedenen Gesellschaftsbereichen ausgeübt. Die Darstellung freiwilligen Engagements nach gesellschaftlichen Bereichen zeigt die Fülle an Möglichkeiten, sich freiwillig zu engagieren. Zivilgesellschaftliche Organisationen erhalten damit einen Überblick über die Engagierten in den jeweiligen Bereichen. Personengruppen, die sich eher unterdurchschnittlich in einem Bereich engagieren, könnten durch gezielte Maßnahmen angesprochen werden. Unterschiede in der Beteiligung in den einzelnen Bereichen gibt es beispielsweise nach Geschlecht, Alter oder Bildung (Vogel et al. 2017a). Im Freiwilligensurvey (FWS) wird freiwilliges Engagement über die Beteiligung in 14 gesellschaftlichen Bereichen erfragt. Es lassen sich deshalb nicht nur allgemeine, sondern auch bereichsspezifische Engagementquoten darstellen. In diesem Abschnitt wird anhand des FWS aufgezeigt, in welchen Bereichen sich die Menschen ab 14 Jahren in Deutschland in den letzten zwölf Monaten freiwillig engagiert haben. Anschließend werden Ergebnisse zum Bereich Sport aus dem aktuellen Sportentwicklungsbericht 2015/2016 in den Blick genommen.

2 Dieses Unterkapitel wurde verfasst von Corinna Kausmann, DZA; Boris Rump, DOSB; Nadiya Kelle, DZA und Julia Simonson, DZA. 
Engagement in 14 Gesellschaftsbereichen (FWS 2014): Der Bereich Sport und Bewegung weist im Vergleich der 14 Bereiche mit insgesamt 16,3 Prozent die höchste Engagementbeteiligung auf (Abb. 4.2). 9,1 Prozent der Menschen engagieren sich freiwillig im Bereich Schule oder Kindergarten, beispielsweise als Eltern- oder SchülervertreterIn. Eine ähnliche bereichsspezifische Engagementquote (9,o Prozent) zeigt sich für den Bereich Kultur und Musik. Im sozialen Bereich, beispielsweise in Wohlfahrtsverbänden oder auch Hilfsorganisationen, sind 8,5 Prozent der Menschen freiwillig engagiert und im kirchlichen oder religiösen Bereich 7,6 Prozent. Der anteilig kleinste gesellschaftliche Bereich des freiwilligen Engagements ist der der Justiz oder Kriminalitätsprobleme. Hier sind o,5 Prozent der Menschen engagiert, zum Beispiel als SchöffInnen oder als BetreuerInnen für Straffällige oder Verbrechensopfer.

- Entwicklung des Engagements in 14 Gesellschaftsbereichen von 1999 bis 2014 (FWS): Es gibt gesellschaftliche Bereiche, die seit 1999 ein deutliches Wachstum an den Anteilen freiwillig Engagierter erfahren haben. Dies trifft vor allem auf die Gebiete Bildung und Soziales zu. Die Anteile im Gesellschaftsbereich Schule oder Kindergarten sind innerhalb der 15 Jahre von 5,9 Prozent auf 9,1 Prozent angestiegen. Im Bereich Kultur und Musik gab es einen Anstieg um 4,1 Prozentpunkte (von 4,9 Prozent auf 9,o Prozent) und im sozialen Be-

Abbildung 4.2 Anteile Engagierter in gesellschaftlichen Bereichen 2014 (in Prozent)

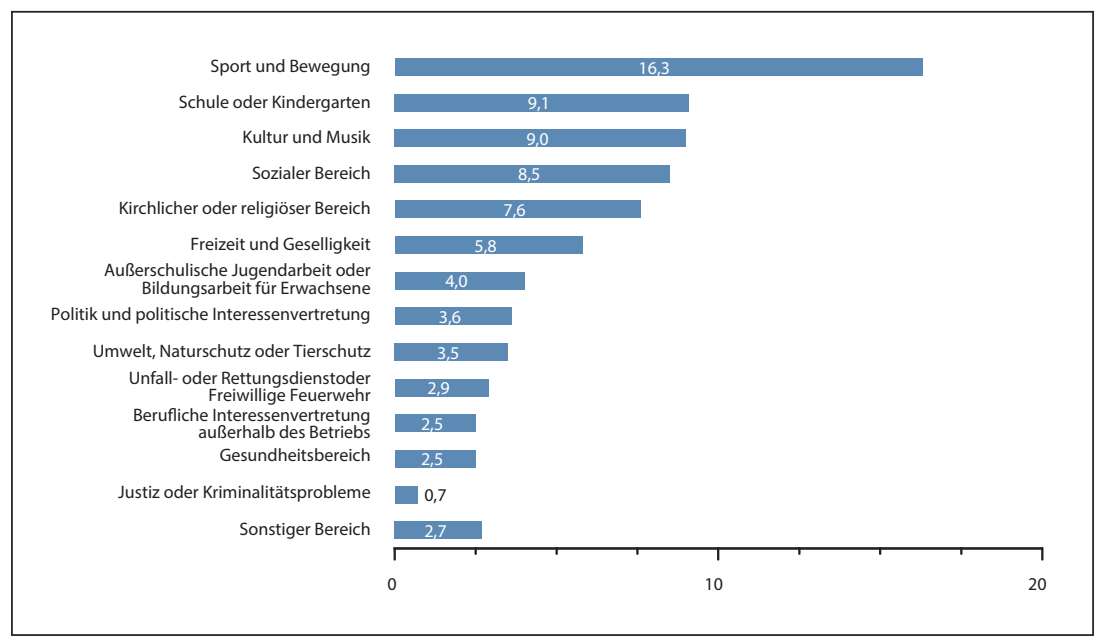

Quelle: FWS 2014, gewichtet, Berechnungen DZA. Basis: Alle Befragten ( $n=28689$ ). 
reich um 4,4 Prozentpunkte (von 4,1 Prozent auf 8,5 Prozent). Bei der Jugendarbeit und Bildungsarbeit für Erwachsene hat sich der Anteil freiwillig Engagierter seit 1999 mehr als verdoppelt: von 1,7 Prozent auf 4,o Prozent.

- Unterschiede im Engagement in 14 Gesellschaftsbereichen für verschiedene Bevölkerungsgruppen (FWS 2014): Im Bereich Sport und Bewegung sind sowohl Frauen als auch Männer am häufigsten freiwillig engagiert, jedoch übersteigt die Anzahl der Männer die der Frauen (siehe Abschnitt 4.5). Auch Altersgruppenunterschiede lassen sich identifizieren: So sind die jüngeren Personen im Alter von 14 bis 29 Jahren anteilig häufig im Bereich Sport und Bewegung oder auch im Bereich Unfall- oder Rettungsdienst bzw. freiwillige Feuerwehr tätig. Bei den 30- bis 49-Jährigen ist der Bereich Schule oder Kindergarten recht bedeutend. Die Personen ab 65 Jahren sind wiederum überdurchschnittlich im sozialen Bereich freiwillig engagiert.

Die skizzierten Merkmale und Entwicklungen für das ehrenamtliche und freiwillige Engagement im Bereich Sport und Bewegung lassen sich auch in den Daten des Sportentwicklungsberichts (SEB) erkennen. Insgesamt engagieren sich in den Sportvereinen rund 1,7 Millionen Mitglieder in ehrenamtlichen Positionen. Dabei werden 1,2 Millionen Positionen von Männern und 0,5 Millionen Positionen von Frauen besetzt. 23 Prozent der Sportvereinsmitglieder engagieren sich zusätzlich als freiwillige HelferInnen bei entsprechenden Arbeitseinsätzen, was hochgerechnet rund 6,3 Millionen Mitglieder sind. Addiert man die Freiwilligen zu den Personen mit einer ehrenamtlichen Position hinzu, so ergibt dies eine Gesamtzahl von rund acht Millionen ehrenamtlich und freiwillig Engagierten in den Sportvereinen. Im Vergleich zum Jahr 2013 zeigen sich keine signifikanten Veränderungen bei der Anzahl der Ehrenamtlichen. Die Zahlen sind auch für alle Ebenen relativ stabil. Dennoch stellt die Bindung und Gewinnung von ehrenamtlichen FunktionsträgerInnen nach wie vor das größte Problem für die Sportvereine dar (vgl. Breuer und Feiler 2017). Daher wird in der Untersuchung 2015/2016 herausgestellt, dass die Ehrenamtsförderung sowie ein angebotsübergreifendes Gemeinwohlmoment zentrale Aufgaben für die Sportvereine darstellen. Dies gilt sowohl für die Vorstandsebene als auch für die Ausführungsebene (Positionen und Funktionen außerhalb des Vorstands wie zum Beispiel TrainerInnen, ÜbungsleiterInnen oder Schieds- und KampfrichterInnen).

Die Beteiligung in den 14 gesellschaftlichen Bereichen zeigt die Vielfalt auf, in der freiwilliges Engagement stattfinden kann. Daraus ergibt sich jedoch auch, dass es verschiedenste Maßnahmen zur Förderung von Engagement und Engagierten geben muss, um dieser Vielfalt und den jeweiligen Anforderungen gerecht werden zu können. Diese Anforderungen können sich zum einen durch thematische Un- 
terschiede ergeben, zum anderen aber auch durch die unterschiedlichen Bevölkerungsgruppen, die in den jeweiligen Bereichen mehr oder weniger engagiert sind. Die Ergebnisse lassen außerdem darauf schließen, dass sich die jeweilige Lebensphase darauf auswirkt, ob und in welchem Bereich sich Menschen beteiligen. Die Ergebnisse aus den Sportentwicklungsberichten zeigen, dass die Sportvereine es trotz vieler Veränderungen in der Gesellschaft (zum Beispiel demografischer Wandel, veränderte Wertorientierungen und Erwartungen an ein Engagement) bisher überwiegend geschafft haben, das ehrenamtliche und freiwillige Engagement in den letzten Jahren stabil zu halten. Allerdings scheint es auch in diesem Bereich entscheidend, die Aufgabe der Bindung und Gewinnung mehr denn je in den Fokus der Vereinsarbeit zu rücken sowie neue Wege in der Ehrenamtsförderung zu gehen.

\subsection{Engagement in den Bundesländern ${ }^{3}$}

Freiwilliges Engagement ist in vielen Fällen ortsgebunden. Daher sind regionale Kontexte und Beschaffenheiten von Bedeutung. Die Auswirkungen des demografischen Wandels können sich in den Ländern oder Regionen ganz unterschiedlich gestalten. Auch die ökonomische Situation einer Region kann das Engagement prägen: Die wirtschaftliche Stärke von Regionen wirkt sich beispielsweise auf Angebotsstrukturen wie die Vereinsdichte oder die Unterstützung von Projekten aus. Ebenso resultieren aus der wirtschaftlichen Stärke auch unterschiedliche Bedarfe, zum Beispiel bezüglich der Grundversorgung oder der Infrastruktur. Die Beschaffenheit der allgemeinen Infrastruktur kann sich ebenfalls förderlich oder hemmend auf eine Beteiligung im Engagement auswirken (Kausmann et al. 2017a).

Engagement nach Bundesländern (FWS 2014): Im Freiwilligensurvey 2014 sind die höchsten Engagementquoten mit einem Anteil von 48,3 Prozent in RheinlandPfalz und von 48,2 Prozent in Baden-Württemberg zu finden (Abb. 4.3). Ebenso gibt es eine überdurchschnittliche Engagementbeteiligung (43,6 Prozent) in den Bundesländern Bayern (47,3 Prozent), dem Saarland (46,4 Prozent), Niedersachsen (46,2 Prozent) und Hessen (44,4 Prozent). 42,7 Prozent der Menschen in Schleswig-Holstein sind freiwillig engagiert und 41,o Prozent in Nordrhein-Westfalen. Die Anteile der Personen in den drei Stadtstaaten, die sich freiwillig engagieren, sind sehr unterschiedlich: Während in Bremen 42,3 Prozent engagiert sind, sind es in Berlin 37,2 Prozent und in Hamburg 36,o Prozent. In den Bundesländern

3 Dieses Unterkapitel wurde verfasst von Corinna Kausmann, DZA; Nadiya Kelle, DZA und Julia Simonson, DZA. 
Abbildung 4.3 Anteile freiwillig Engagierter in den 16 Ländern 2014 (in Prozent)

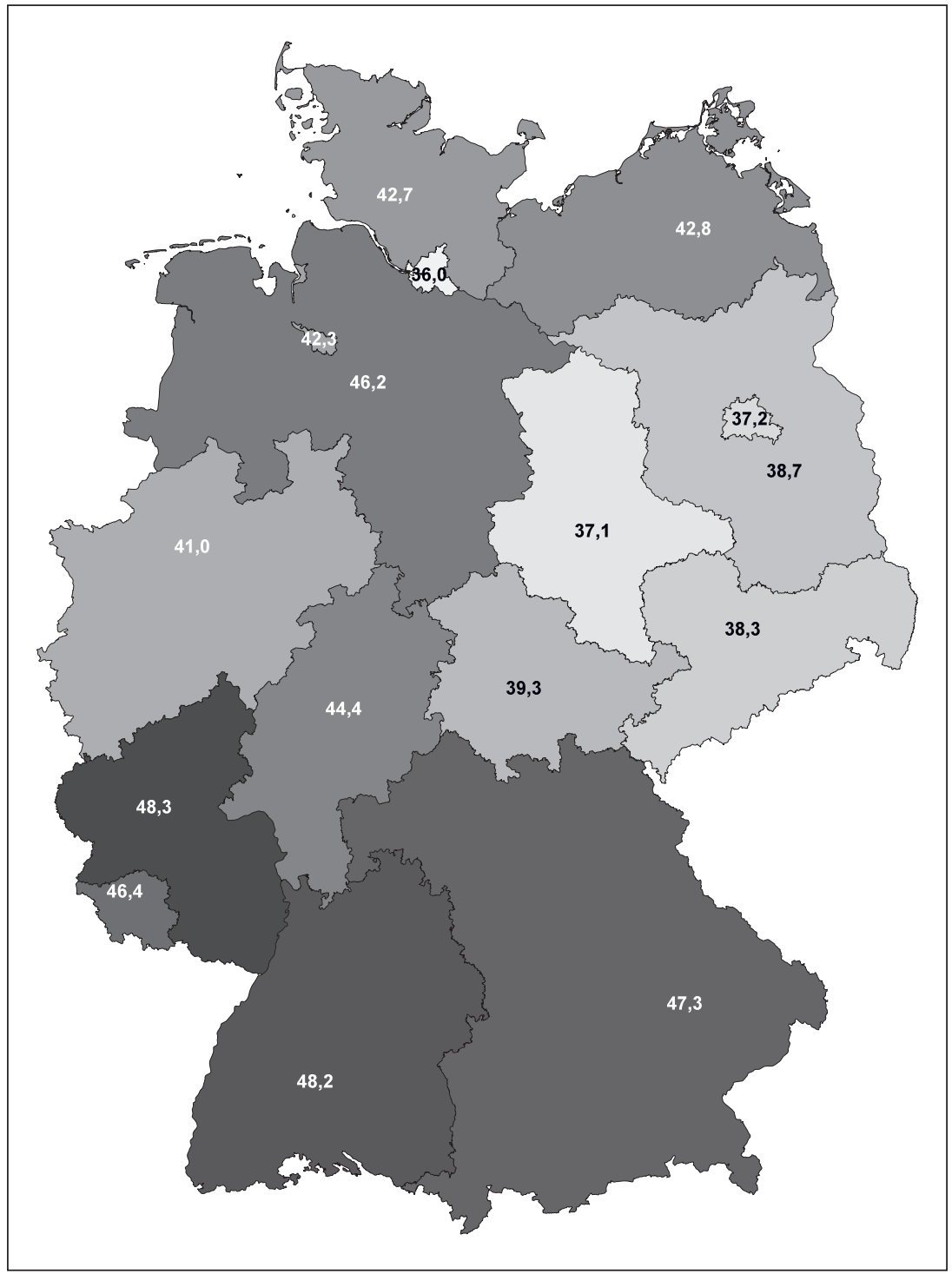

Quelle: FWS 2014, gewichtet, Berechnungen DZA. Basis: Alle Befragten ( $n=28689$ ). Darstellung DZA, Karte erstellt mit Easymap 
im Osten Deutschlands sind anteilig die meisten Personen in Mecklenburg-Vorpommern (42,8 Prozent) freiwillig engagiert. Die Engagementbeteiligung in Thüringen liegt bei 39,3 Prozent, in Brandenburg bei 38,7 Prozent, in Sachsen sind es 38,3 Prozent und in Sachsen-Anhalt 37,1 Prozent.

Das freiwillige Engagement in den 16 Bundesländern unterscheidet sich somit nicht nur zwischen Ost- und Westdeutschland. Die Beteiligung ist in den westdeutschen Flächenländern höher als in den neuen Bundesländern sowie in den drei Stadtstaaten. Die Ergebnisse weisen auch ein Nord-Süd-Gefälle auf, bei dem die Beteiligung im freiwilligen Engagement im Süden höher ausfällt als im Norden Deutschlands. In Bezug auf den Zusammenhang zwischen der wirtschaftlichen Stärke und der Engagementquote zeigt sich, dass die südlich gelegenen Länder wie Baden-Württemberg, Bayern oder Hessen, die derzeit zu den finanzstarken Ländern zählen, die höchsten Engagementquoten aufweisen. Zugleich weisen finanzschwächere Länder, zu denen Thüringen, Brandenburg, Sachsen und Sachsen-Anhalt zählen, geringere Engagementquoten auf (Statistisches Bundesamt 2016). In finanzschwächeren Ländern ist die Arbeitslosigkeit höher und niedrigere Einkommen sind weiter verbreitet als in finanzstärkeren, was die Beteiligung im freiwilligen Engagement beeinträchtigen kann (Simonson und Hameister 2017). Außerdem verfügen finanzschwächere Länder über weniger finanzielle Mittel, um beispielsweise eine notwendige Infrastruktur für freiwilliges Engagement zu fördern.

Weiterhin zeigen sich Unterschiede in den Anknüpfungspunkten durch die Organisationsformen des freiwilligen Engagements. So ist die Vereinsdichte im Osten Deutschlands geringer als im Westen (Zivilgesellschaft in Zahlen, ZiviZ 2012). Dabei bilden Vereine und Verbände die Organisationsform, in der Engagement am häufigsten ausgeübt wird (siehe Abschnitt 4.8). Zudem sind Menschen in den ostdeutschen Ländern anteilig seltener Mitglied in einer kirchlichen oder religiösen Vereinigung, die Anstöße zum Engagement geben können sowie auch Ressourcen zur Verfügung stellen, um Engagement zu ermöglichen und zu fördern.

\subsection{Engagement und Altersstrukturen ${ }^{4}$}

Die Beteiligung im freiwilligen Engagement unterscheidet sich zwischen verschiedenen Altersgruppen. Je nach Lebensphase variieren die Anknüpfungspunkte für eine freiwillige Tätigkeit genauso wie die Hemmnisse, eine freiwillige Tätigkeit

4 Dieses Unterkapitel wurde verfasst von Corinna Kausmann, DZA; Nadiya Kelle, DZA und Julia Simonson, DZA. 
aufzunehmen. Weiterhin geht der demografische Wandel mit einer gestiegenen Lebenserwartung und einer durch den Geburtenrückgang hervorgerufenen Alterung der Gesellschaft einher. Damit sind auch Veränderungen für das freiwillige Engagement denkbar. So könnten Netzwerkhilfe oder auch freiwilliges Engagement die sich wandelnden und wachsenden Bedarfe Älterer nach Unterstützung ausgleichen. Da die gestiegene Lebenserwartung auch mit einer längeren Ruhestandsphase - die oft in guter Gesundheit und wirtschaftlicher Sicherheit verbracht wird - einhergeht, sind Ältere nicht nur EmpfängerInnen, sondern auch Ausführende eines freiwilligen Engagements.

Engagement in verschiedenen Altersgruppen (FWS): Im Freiwilligensurvey 2014 ist es aufgrund der offenen Altersabfrage möglich, verschiedene Altersgruppen zu betrachten. In Abbildung 4.4 wird die Engagementbeteiligung differenziert nach Fünf-Jahres-Altersgruppen dargestellt. Die anteilig höchste Engagementbeteiligung ist bei den jungen Menschen im Alter von 14 bis 19 Jahren auszumachen über die Hälfte dieser Altersgruppe ist freiwillig engagiert (Abb. 4.4).

Ebenfalls eine recht hohe Beteiligung zeigt sich in der Gruppe der 20- bis 24-Jährigen mit 48,4 Prozent. Bei den darauffolgenden Altersgruppen, den 25- bis 29-Jährigen und den 30- bis 34-Jährigen, zeigen sich mit 40,1 und 41,2 Prozent deutlich geringere Anteile freiwillig Engagierter. In den Altersgruppen zwischen 35 und 49 Jahren sind die Anteile Engagierter vergleichsweise einheitlich und liegen zwischen 47,5 und 49,8 Prozent. Im Alter zwischen 50 und 69 sind die Antei-

Abbildung 4.4 Anteile freiwillig engagierter Personen 2014, nach Fünf-Jahres-Altersgruppen (in Prozent)

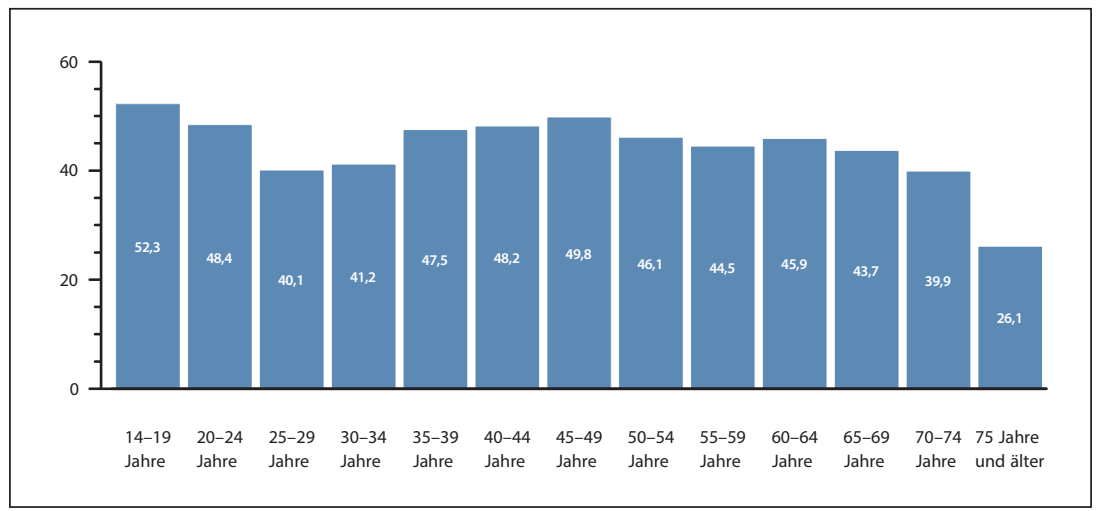

Quelle: FWS 2014, gewichtet, Berechnungen DZA. Basis: Alle Befragten $(n=28689)$. 
le hingegen etwas geringer und liegen zwischen 43,7 und 45,9 Prozent. Deutlich geringere Anteile sind im Alter von 70 bis 74 Jahren mit 39,9 Prozent und bei den 75-Jährigen und Älteren mit 26,1 Prozent auszumachen.

Auch wenn die Anteile freiwillig Engagierter in den höheren Altersgruppen geringer sind als die der Jüngeren, zeigt sich, dass die Engagementbeteiligung älterer Menschen in den vergangenen 15 Jahren deutlich angestiegen ist (Vogel et al. 2017b). Dabei ist ein großer Anstieg bei den 65- bis 74-Jährigen festzustellen. Während im Jahr 1999 26,5 Prozent in dieser Altersgruppe freiwillig engagiert waren, waren es in 2014 41,5 Prozent. Und auch das Engagement der über 75-Jährigen ist innerhalb der 15 Jahre angestiegen - um 9,5 Prozentpunkte von 16,6 Prozent auf 26,1 Prozent.

Die unterschiedlichen Anteile freiwilligen Engagements lassen auf einen Einfluss der jeweiligen Lebensphase auf die Beteiligung im freiwilligen Engagement schließen. Während in den jüngeren Altersgruppen die Personen möglicherweise noch mehr Zeit für Freizeitaktivitäten haben, ist in den älteren Gruppen, durch den Einstieg in die Berufstätigkeit oder auch die Gründung einer Familie, der zeitliche Rahmen stärker begrenzt. Die Beteiligung im Engagement bei Personen, die sich im Ruhestand befinden, ist nur geringfügig kleiner als in den Altersgruppen unter 65 Jahren. In den höheren Altersgruppen sind die Anteile freiwillig Engagierter deutlich geringer als im jüngeren oder mittleren Lebensalter, was beispielsweise auf gesundheitliche Einschränkungen oder Altersgrenzen zurückzuführen sein kann. Doch die Anteile der Beteiligung im Engagement von Älteren sind in den letzten 15 Jahren ebenfalls deutlich angestiegen. In Anbetracht dieser gestiegenen Beteiligung soll im Folgenden das Engagement älterer Menschen noch etwas genauer beleuchtet werden.

Engagement von älteren Menschen (DEAS): Ältere Engagierte richten ihre freiwillige Tätigkeit am häufigsten an ältere Menschen (Hagen und Simonson 2017). Aus diesem Grund wird im Folgenden mit Hilfe der Daten des Deutschen Alterssurveys (DEAS) 2014 das Engagement von Älteren in Seniorengruppen analysiert. Dabei handelt es sich beispielsweise um Seniorenselbsthilfegruppen, Seniorengenossenschaften, Weiterbildungsgruppen oder auch Sport- und Tanzgruppen. Zusätzlich zum ehrenamtlichen Engagement in Seniorengruppen soll auch die Neuaufnahme des Engagements im höheren Alter aufgezeigt werden. Es wird dargestellt, wer ein organisationsgebundenes ehrenamtliches Engagement in den letzten sechs Kalenderjahren aufgenommen hat und 2014 immer noch ausübt. Dies geschieht auf Basis aller Befragten. Zusätzlich ist es auch möglich darzulegen, wie sich die Neu-Beteiligung anteilig zum Engagement insgesamt verhält. Das Engagement in Seniorengruppen wird nur bei Personen ab 50 Jahren erfragt. Die Neuaufnahme eines Engagements wird bereits bei Personen ab 40 Jahren erfasst. 
- Engagement in Seniorengruppen im DEAS: Laut DEAS sind im Jahr 2014 insgesamt 5,6 Prozent der Befragten im Alter von 50 bis 85 Jahren ehrenamtlich in einer Seniorengruppe engagiert (Abb. 4.5). Die Aufnahme eines Ehrenamtes bzw. einer Funktion in einer Seniorengruppe stellt sich über die Altersgruppen unterschiedlich dar: Bis zu einem Alter von 65 Jahren zeigen sich keine Altersgruppenunterschiede für die Beteiligung in Seniorengruppen. Der Anteil Engagierter in der Gruppe der 66- bis 71-Jährigen ist mit 8,6 Prozent am höchsten. Das seniorenspezifische Engagement von Männern ab 50 Jahren unterscheidet sich nicht von dem der Frauen ab 50 Jahren.

Rund ein Viertel (25,4 Prozent) der Personen, die ganz allgemein als ehrenamtlich engagiert gelten, engagieren sich in Seniorengruppen. Dieser Anteil von Engagierten, die in Seniorengruppen tätig sind, ist in den höheren Altersgruppen größer: 15,8 Prozent der ehrenamtlich Engagierten im Alter von 54 bis 59 Jahren sind in solch einer Gruppe tätig, wohingegen es bei den 78 - bis 83-jährigen Engagierten 51,4 Prozent sind.

- Neuaufnahme eines Engagements im DEAS: Um die Neuaufnahme eines Engagements zu erfassen, wird betrachtet, wer innerhalb der letzten sechs Kalenderjahre (also zwischen 2009 und 2014) eine ehrenamtliche Tätigkeit aufgenommen hat. Insgesamt haben 7,1 Prozent der Personen zwischen 40 und 85 Jahren in dieser Zeitspanne mindestens eine ehrenamtliche Tätigkeit aufgenommen (Abb. 4.6). Von allen ehrenamtlich engagierten Personen im Jahr 2014 hat somit ein Drittel ein neues Engagement in den letzten sechs Jahren begonnen und zwei Drittel sind bereits länger ehrenamtlich engagiert. $\mathrm{Zu}$ beachten ist, dass hier das zuletzt neu aufgenommene Engagement analysiert wird. Deshalb ist es bei der Betrachtung des Neu-Engagements möglich, dass die Neu-Engagierten bereits ein anderes Engagement ausüben und die Anteile von Personen, die ein Engagement in den letzten sechs Jahren begonnen haben und vorher nicht ehrenamtlich engagiert waren, geringer sind.

Die Anteile der jüngeren Altersgruppen, die in den letzten sechs Jahren ein ehrenamtliches Engagement aufgenommen haben, sind höher als die der älteren Gruppen. Jeweils über 7 Prozent der Personen im Alter zwischen 48 und 70 Jahren engagieren sich in einer neuen Tätigkeit (Abb. 4.6). Der höchste Anteil an Personen, die eine neue Funktion oder ein neues Ehrenamt aufgenommen haben, ist mit 11,o Prozent bei den 42- bis 47-Jährigen zu finden. $\mathrm{Ab}$ dem Alter von 72 Jahren werden die Anteile deutlich geringer und liegen bei 3,4 (72- bis 77-Jährige) bzw. 1,3 (78- bis 83-Jährige) Prozent.

Das Verhältnis von Neu-Engagierten an allen ehrenamtlich Engagierten stellt sich für die Altersgruppen wie folgt dar: Etwas über die Hälfte (51,5 Prozent) der Engagierten im Alter von 42 bis 47 Jahren haben mindestens eine ihrer Tätigkeiten in den letzten sechs Jahren aufgenommen. Von den ehrenamtlich 
Abbildung 4.5 Anteile der ehrenamtlich Engagierten in Seniorengruppen, nach Alter und Geschlecht, im Jahr 2014 (in Prozent)

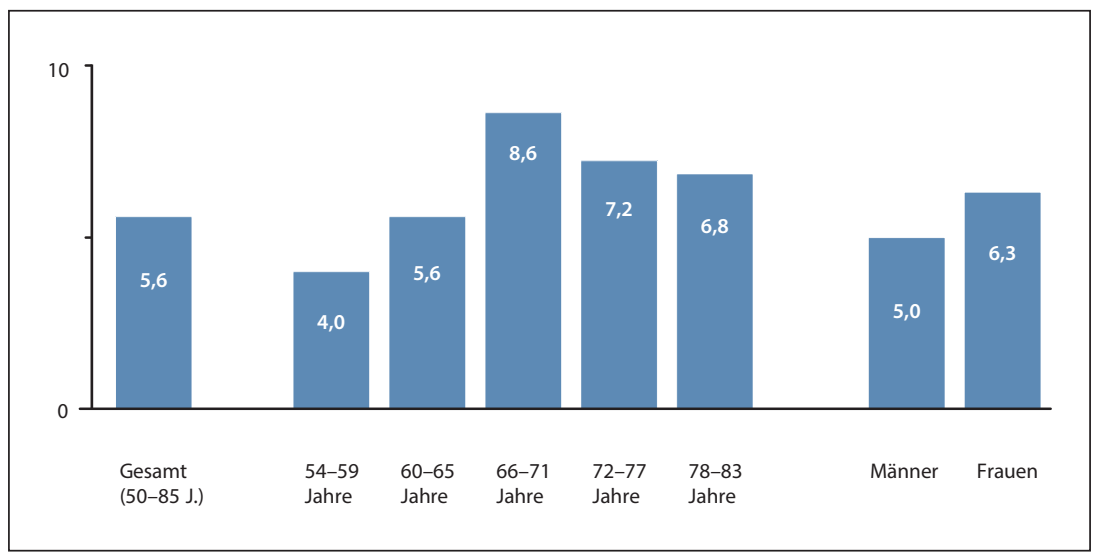

Quelle: DEAS 2014, gewichtet, Berechnungen DZA. Basis: Befragte zwischen 50 und 85 Jahren $(n=4952)$.

Abbildung 4.6 Anteile der Personen, die in den letzten sechs Kalenderjahren ein Engagement aufgenommen haben, gesamt und nach Alter, im Jahr 2014 (in Prozent)

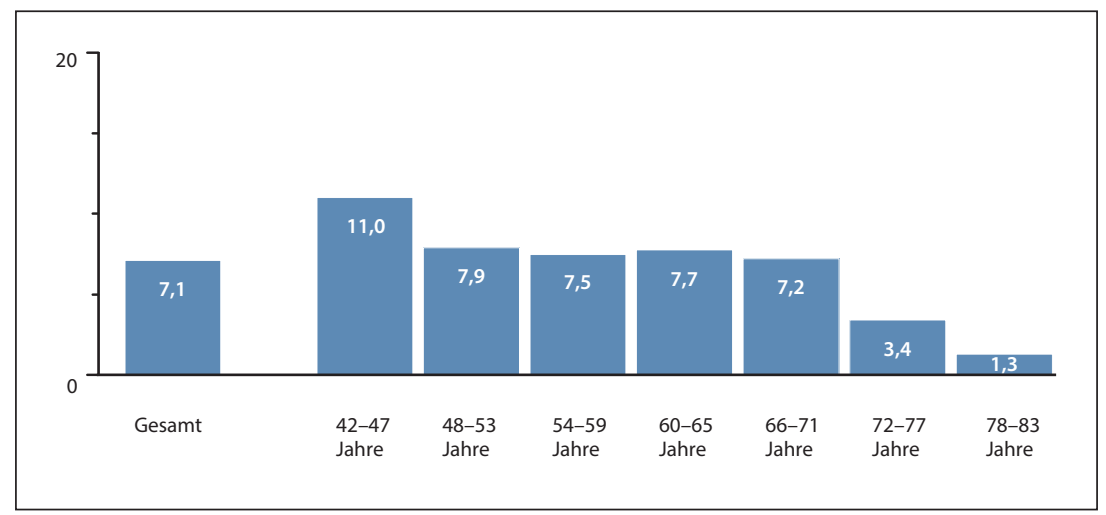

Quelle: DEAS 2014, gewichtet, Berechnungen DZA. Basis: Alle Befragten $(n=5993)$. 
Engagierten im Alter von 72 bis 77 Jahren sind es 18,6 Prozent und von denjenigen im Alter von 78 bis 83 Jahren 9,4 Prozent.

Das Engagement von Menschen im höheren Lebensalter ist vor allem auch durch die bessere Gesundheit im Alter geprägt (Wolff et al. 2017). Eine längere und bei besserer Gesundheit verbrachte Ruhestandsphase begünstigt Engagement, zum Beispiel in einer Seniorengruppe. Ein Viertel aller älteren Engagierten hat eine Funktion oder ein Ehrenamt in einer Seniorengruppe. Die höchsten Anteile Engagierter in solch einer Gruppe sind in den ersten Jahren des Ruhestandes zu finden. Das lässt darauf schließen, dass Seniorengruppen zum Teil den wegfallenden Anknüpfungspunkt der Erwerbstätigkeit ausgleichen können. Die Neuaufnahmen einer ehrenamtlichen Tätigkeit werden erst ab 70 Jahren geringer. Engagierte in einer späteren Lebensphase weisen jedoch einen geringeren Anteil an Neu-Engagierten auf als engagierte Personen mittleren Alters. Das deutet darauf hin, dass die Älteren einem einmal bestehenden Engagement konstanter erhalten bleiben als Jüngere.

\subsection{Engagement und Geschlecht ${ }^{5}$}

Die gesellschaftliche Arbeitsteilung, die Sozialisation oder auch die strukturellen Rahmenbedingungen können sich auf verschiedenste Weise auf die Engagementbeteiligung sowie die Ausgestaltung des Engagements von Frauen und Männern auswirken (Vogel et al. 2017d). Im Zuge der Bildungsexpansion, der gestiegenen Erwerbsbeteiligung von Frauen oder auch sich wandelnder Familienstrukturen können sich die geschlechtsspezifischen Unterschiede verstärken oder abschwächen. Zudem können sich je nach Lebensphase Motive, Ressourcen sowie Netzwerke von Frauen und Männern unterscheiden.

Engagement von Frauen und Männern in verschiedenen Altersgruppen (FWS 2014): Im Deutschen Freiwilligensurvey 2014 zeigen sich deutliche Differenzen in der Beteiligung im freiwilligen Engagement von Frauen und Männern. In der Gesamtbetrachtung der Personen ab 14 Jahren sind Männer mit 45,7 Prozent anteilig häufiger freiwillig engagiert als Frauen (41,5 Prozent). Die Engagementquoten nach Altersgruppen zeigen verschiedenartige Geschlechterunterschiede auf (Abb. 4.7).

5 Dieses Unterkapitel wurde verfasst von Corinna Kausmann, DZA; Nadiya Kelle, DZA und Julia Simonson, DZA. 
Abbildung 4.7 Anteile freiwillig engagierter Frauen und Männer 2014, nach Altersgruppen (in Prozent)

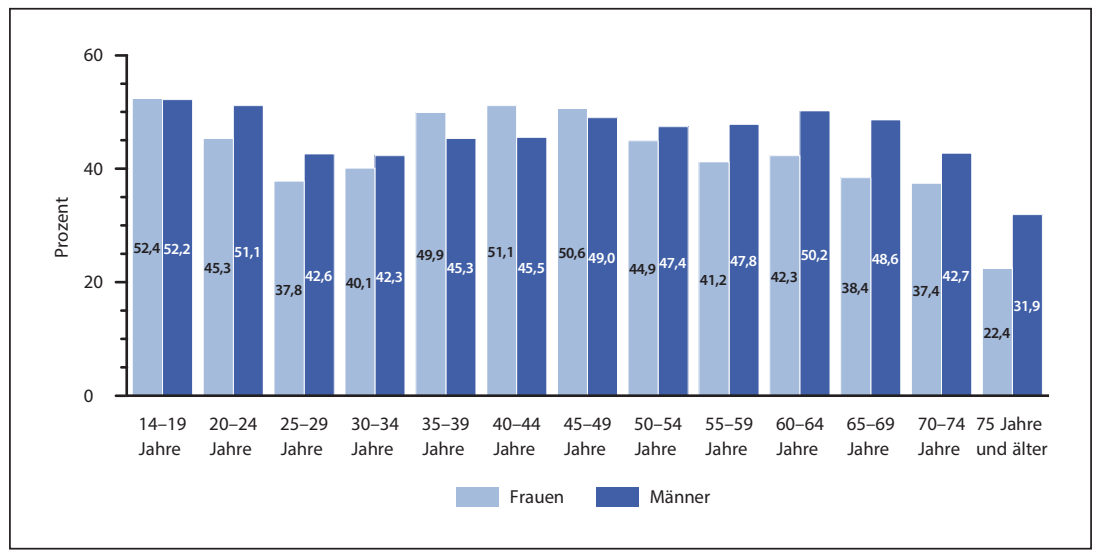

Quelle: FWS 2014, gewichtet, Berechnungen DZA. Basis: Alle Befragten $(n=28689)$.

Im höheren Lebensalter sind wiederum höhere Engagementraten für Männer $\mathrm{zu}$ verzeichnen. So sind in der Gruppe der Personen im Alter von 65 bis 69 Jahren 48,6 Prozent der Männer freiwillig engagiert und lediglich 38,4 Prozent der Frauen. Eine ähnlich große Differenz in der Beteiligung zeigt sich für 75-Jährige und Ältere: 31,9 Prozent der Männer sind engagiert und 22,4 Prozent der Frauen. Aber auch in den jüngeren Lebensjahren zeigen sich diese Differenzen, wenn auch auf einem etwas geringeren Niveau, wie beispielsweise bei den 25- bis 29-Jährigen oder den 30- bis 34-Jährigen (Abb. 4.7). Doch es gibt auch Altersgruppen, in denen diese Geschlechterunterschiede hinsichtlich der Engagementbeteiligung nicht vorliegen. In den jüngsten Altersgruppen im Freiwilligensurvey 2014 sind jeweils über die Hälfte der Frauen sowie der Männer freiwillig engagiert. Aber auch im mittleren Alter ( 35 bis 39 Jahre, 40 bis 44 Jahre und 45 bis 49 Jahre) unterscheiden sich die Anteile freiwillig Engagierter nicht statistisch signifikant nach Geschlecht.

Engagement von Frauen und Männern in verschiedenen Gesellschaftsbereichen (FWS 2014): Nicht nur bei der Beteiligung am freiwilligen Engagement liegen Geschlechterunterschiede vor, sondern auch bei der Ausgestaltung des Engagements. Frauen und Männer ab 14 Jahren in Deutschland engagieren sich zu unterschiedlichen Anteilen in den verschiedenen gesellschaftlichen Bereichen. Sowohl Frauen als auch Männer sind im Vergleich der 14 gesellschaftlichen Bereiche des freiwil- 
ligen Engagements anteilig am häufigsten im Bereich Sport und Bewegung freiwillig engagiert. Hier findet sich jedoch auch die größte geschlechtsspezifische Differenz mit 6,5 Prozentpunkten: 19,6 Prozent der Männer und 13,1 Prozent der Frauen engagieren sich im Sport. Männer sind ebenfalls anteilig häufiger in den Bereichen Unfall- oder Rettungsdienst und freiwillige Feuerwehr sowie in der Politik und der politischen Interessenvertretung engagiert als Frauen. Die Anteile der Frauen sind wiederum im kirchlichen oder religiösen Bereich, im sozialen Bereich sowie im Bereich Schule oder Kindergarten höher als die der Männer (Kausmann et al. 2017b).

Die Ergebnisse des Freiwilligensurveys 2014 weisen darauf hin, dass die unterschiedliche Engagementbeteiligung von Frauen und Männern nach Lebensphasen und deren typischen Lebenssituationen variiert. In jüngeren Jahren, die von ähnlicher schulischer oder beruflicher Ausbildung geprägt sind, finden sich diese Geschlechterunterschiede nicht. Typischerweise lässt sich das Alter von 25 bis 34 Jahren durch den Einstieg und das Festigen der Erwerbsarbeit sowie auch durch eine mögliche Familiengründung charakterisieren. Hier zeigen sich zum Teil deutliche Differenzen im Engagement von Frauen und Männern, ebenso wie in der Phase des Ruhestandes. Diese Unterschiede können sich zum einen beispielsweise auf verschiedene Vereinbarkeitsmuster oder auch Rollenvorstellungen zurückführen lassen (Kausmann et al. 2017b). Zum anderen zeigt sich jedoch auch in der Altersstruktur Deutschlands, dass zum Beispiel ältere Frauen anteilig seltener freiwillig engagiert sind als ältere Männer, jedoch die absolute Anzahl der freiwillig Engagierten ungefähr gleich groß ist, da es in Deutschland mehr ältere Frauen als ältere Männer gibt (Vogel et al. 2017b). Die Bereiche, in denen das Engagement ausgeübt wird, lassen darauf schließen, dass sich die bestehende geschlechtsspezifische Arbeitsteilung auch im freiwilligen Engagement niederschlägt. Frauen sind dabei eher in familienbezogenen und sozialen Bereichen freiwillig tätig. Bei ihnen scheint das freiwillige Engagement auch viel stärker durch die Lebensphasen geprägt zu sein als bei Männern.

\subsection{Engagement und Migrationshintergrund ${ }^{6}$}

Die Entwicklung der Zuwanderung nach Deutschland der letzten Jahre hat verdeutlicht, dass Bedarfe an freiwilligem Engagement steigen. Sie hat aber auch Potenziale aufgezeigt, die sich daraus entwickeln können. Denn Personen mit Migra-

6 Dieses Unterkapitel wurde verfasst von Corinna Kausmann, DZA; Nadiya Kelle, DZA und Julia Simonson, DZA. 
tionshintergrund sind nicht nur EmpfängerInnen des freiwilligen Engagements, sondern auch freiwillig Engagierte. Je nach Herkunftsland, Kultur oder Migrationsgeschichte der MigrantInnen ergeben sich unterschiedliche Muster in der Ausübung freiwilligen Engagements beziehungsweise in der benötigten Unterstützung.

Engagement nach Migrationshintergrund (FWS 2014): Im Freiwilligensurvey 2014 werden verschiedene Formen des Migrationshintergrundes aufgegliedert. Ganz allgemein zeigt sich für die Wohnbevölkerung Deutschlands ab 14 Jahren, dass Menschen ohne Migrationshintergrund anteilig häufiger freiwillig engagiert sind als Menschen mit Migrationshintergrund (Abb. 4.8). Im Freiwilligensurvey werden neben den Menschen ohne Migrationshintergrund vier Gruppen von Per-

Abbildung 4.8 Anteile freiwillig engagierter Personen 2014, nach Art des Migrationshintergrundes (in Prozent)

Personen ohne

Migrationshintergrund

Personen mit

Migrationshintergrund

$\checkmark$

Ohne eigene Zuwanderungserfahrung, mit Migrationshintergrund

Personen mit

deutscher

Staatsangehörigkeit

Personen ohne

deutsche

Staatsangehörigkeit

\section{6,8}

\section{1,5}

\section{3,2}

31,1

Mit eigener Zuwanderungserfahrung

Personen mit deutscher

Staatsangehörigkeit

Personen ohne deutsche

Staatsangehörigkeit
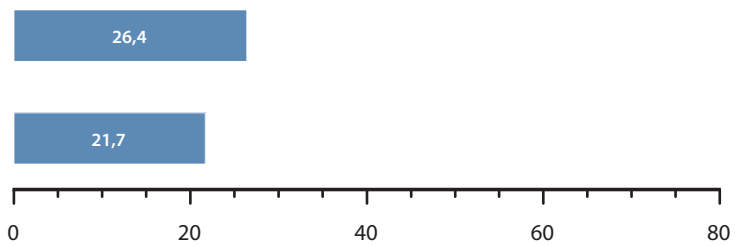

Quelle: FWS 2014, gewichtet, Berechnungen DZA. Basis: Alle Befragten ( $n=28689$ ). 
sonen mit Migrationshintergrund unterschieden. Diese vier Gruppen wurden anhand der Kombination der jeweils zweistufigen Merkmale „Migrationserfahrung" und "Staatsbürgerschaft" gebildet (Migrationserfahrung: ja, MigrantInnen erster Generation bzw. Migrationserfahrung: nein, MigrantInnen zweiter Generation; Staatsbürgerschaft: ja bzw. nein). Unterscheidet man diese Gruppen, so zeigt sich ein differenzierteres Bild: 43,2 Prozent der MigrantInnen ohne eigene Zuwanderungserfahrung, die die deutsche Staatsangehörigkeit besitzen, sind freiwillig engagiert. Das ist nur geringfügig weniger als bei den Personen ohne Migrationshintergrund. Von denjenigen, die ebenfalls keine eigene Zuwanderungserfahrung gemacht haben, jedoch nicht die deutsche Staatsangehörigkeit haben, sind 31,1 Prozent freiwillig engagiert. Der geringste Anteil an freiwillig Engagierten liegt mit 21,7 Prozent bei den Personen, die eine eigene Zuwanderungserfahrung sowie keine deutsche Staatsangehörigkeit haben.

Personen, die in Deutschland sozialisiert wurden, scheinen einen besseren Anschluss an zivilgesellschaftliche Organisationen und Tätigkeitsfeldern zu finden als Personen, die erst später in ihrem Leben nach Deutschland zugewandert sind. So ist die Beteiligung von MigrantInnen der zweiten Generation im freiwilligen Engagement höher als bei den MigrantInnen der ersten Generation. Auch der Einfluss der Staatsangehörigkeit wird deutlich. Die dargestellten Unterschiede bei der Beteiligung im freiwilligen Engagement zwischen Personen ohne und mit Migrationshintergrund sowie zwischen den unterschiedlichen Formen des Migrationshintergrundes ergeben sich ebenfalls aus dem Alter, dem Bildungsstand oder auch dem Einkommen der Personen. Jedoch sind diese Faktoren nicht ausschließlich ausschlaggebend für die unterschiedlichen Beteiligungsquoten. Es können ebenfalls unterschiedliche Kenntnisse über Gelegenheiten zum freiwilligen Engagement vorliegen oder auch kulturelle Unterschiede in Bezug auf die Definition freiwilligen Engagements und der Zuschreibung gewisser Tätigkeiten zu diesem Feld (Vogel et al. 2017c).

\subsection{Engagement nach Bildung und Erwerbsstatus ${ }^{7}$}

Die individuellen Ressourcen sind für den Zugang zu freiwilligem Engagement oftmals ausschlaggebend. Nicht nur materielle Ressourcen wie das Einkommen wirken sich auf eine Beteiligung aus. Auch immaterielle Ressourcen wie beispielsweise die Bildung können sich sowohl fördernd als auch hemmend auf die Aus-

7 Dieses Unterkapitel wurde verfasst von Corinna Kausmann, DZA; Nadiya Kelle, DZA und Julia Simonson, DZA. 
übung einer freiwilligen Tätigkeit auswirken. So haben Personen mit einer höheren Bildung eher einen Zugang zu Informationen über freiwilliges Engagement und mehr Möglichkeiten, für ihre Interessen einzutreten, als Personen mit niedriger Bildung.

Engagement nach Bildungsgruppen (FWS 2014): In Abbildung 4.9 sind die Anteile freiwillig Engagierter in den unterschiedlichen Bildungsgruppen dargestellt. Die Bildungsgruppen basieren im Freiwilligensurvey 2014 auf der Schulbildung. Aufgrund des Bildungsniveaus lässt sich eine klare Verteilung der Engagementbeteiligung erkennen. Die Gruppe der Personen mit niedriger Bildung ist mit 28,3 Prozent anteilig am geringsten freiwillig engagiert. Personen mit einer hohen Schulbildung sind wesentlich häufiger engagiert. Mehr als die Hälfte der Personen dieser Gruppe (52,3 Prozent) üben ein freiwilliges Engagement aus. Der Anteil freiwillig Engagierter ist in der Gruppe der SchülerInnen am höchsten. Bei diesen Personen, die ihre Schulausbildung ja noch nicht abgeschlossen haben, beträgt der Anteil freiwillig Engagierter 54,8 Prozent.

Abbildung 4.9 Anteile freiwillig engagierter Personen 2014, nach Bildung (in Prozent)

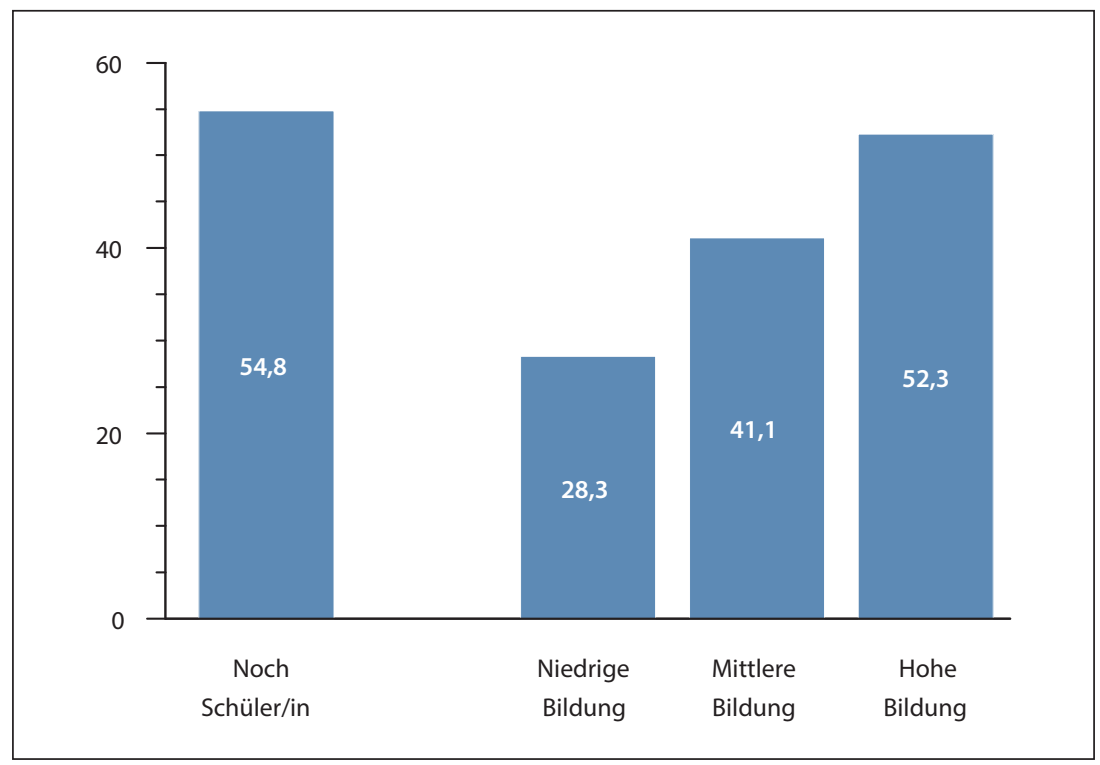

Quelle: FWS 2014, gewichtet, Berechnungen DZA. Basis: Alle Befragten ( $n=28689$ ). 
Engagement nach Erwerbsstatus (FWS 2014): Ebenso wie die Bildung kann auch der Erwerbsstatus als eine Ressource für freiwilliges Engagement dienen. Gleichwohl besteht jedoch die Möglichkeit, dass der Erwerbsstatus einer Person eine hemmende Wirkung auf die Beteiligung im Engagement haben kann. Während eine Erwerbstätigkeit Möglichkeiten und Anstöße zum freiwilligen Engagement liefern kann, kann eben diese Arbeit auch zu einer Verknappung der zur Verfügung stehenden Zeit führen und die Aufnahme einer freiwilligen Tätigkeit erschweren oder verhindern. Auf der anderen Seite besteht die Möglichkeit, dass Personen, die nicht erwerbstätig sind, sich aufgrund einer größeren zur Verfügung stehenden Freizeit eher freiwillig engagieren. Jedoch sind auch bei nichterwerbstätigen Personen hemmende Faktoren aufgrund ihres Erwerbsstatus möglich: Spezifische Gelegenheitsstrukturen sind nicht mehr vorhanden oder die gleichen Ressourcen, die für eine Arbeitsstelle erforderlich sind, fehlen ebenso für die Aufnahme eines Engagements. Personen, die einer Erwerbsarbeit nachgehen, sind anteilig deutlich häufiger freiwillig engagiert als arbeitslos gemeldete Personen. Über die Hälfte derjenigen, die sich in einer Ausbildung befinden, sowie derjenigen, die einer Teilzeitbeschäftigung bzw. einer geringfügigen Beschäftigung nachgehen, sind freiwillig engagiert (Abb. 4.10). Der Anteil Engagierter bei den Vollzeitbeschäftigten ist mit 46,7 Prozent etwas geringer. Arbeitslos Gemeldete weisen im Vergleich mit 21,6 Prozent die geringste Engagementquote auf. Eine unterdurchschnittliche,

Abbildung 4.10 Anteile freiwillig Engagierter 2014, nach Erwerbsstatus (in Prozent)

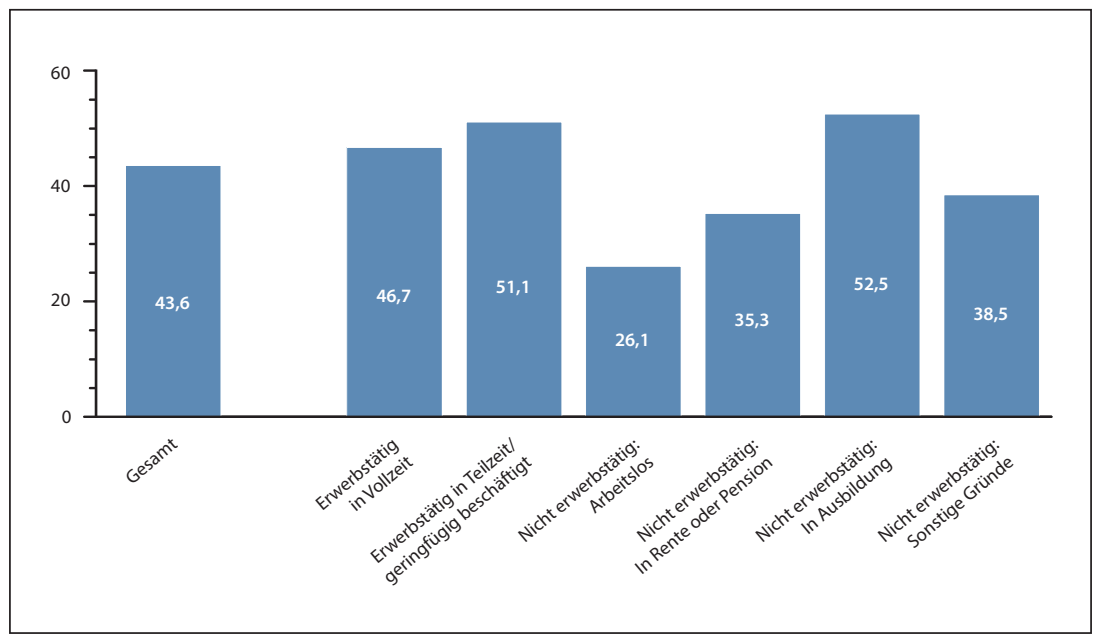

Quelle: FWS 2014, gewichtet, Berechnungen DZA. Basis: Alle Befragten ( $n=28$ 590). 
aber deutlich höhere Quote als die der arbeitslos Gemeldeten haben die freiwillig Engagierten, die aus sonstigen Gründen nicht erwerbstätig sind, sowie Personen im Ruhestand.

Betrachtet man die Engagementquoten differenziert nach der schulischen Bildung beziehungsweise nach dem Erwerbsstatus der Personen, zeigt sich, dass sich Menschen mit einer besseren sozioökonomischen Ressourcenausstattung zu höheren Anteilen engagieren als Personen mit geringeren Ressourcen. Diese Faktoren wirken nicht nur einzeln, sondern auch in Kombination miteinander. So ist die Wahrscheinlichkeit höher, dass Menschen sich freiwillig engagieren, wenn sie erwerbstätig sind und eine hohe Schulbildung haben. Andererseits führt beispielsweise eine Kombination von geringer Bildung und Erwerbslosigkeit zu einer geringeren Wahrscheinlichkeit der Beteiligung im Engagement (Simonson und Hameister 2017).

\subsection{Organisationsgebundenes vs. informelles Engagement ${ }^{8}$}

Freiwilliges Engagement kann in verschiedenen Formen organisiert sein. Dabei stehen sich formal und individuell organisierte Varianten freiwilligen Engagements gegenüber. Im Freiwilligensurvey werden fünf Organisationsformen unterschieden. $\mathrm{Zu}$ den formalen zivilgesellschaftlichen Organisationen zählen hier die Vereine und Verbände, die Kirche oder auch religiöse Vereinigungen, staatliche oder kommunale Einrichtungen sowie andere formal organisierte Einrichtungen. Als fünfte Gruppe werden im Freiwilligensurvey die individuell organisierten Gruppen betrachtet. Dabei handelt es sich beispielsweise um Initiativen, Projekte oder selbstorganisierte Gruppen (Simonson und Vogel 2017).

Organisationsgebundenes und informelles Engagement (FWS 2014): Über die Hälfte der freiwillig engagierten Personen üben, nach dem Freiwilligensurvey 2014, ihre Tätigkeit im Rahmen eines Vereins oder Verbandes aus (Abb. 4.11). In einer Kirche oder religiösen Vereinigung (und damit ebenfalls in einer formal organisierten Einrichtung) sind 13,0 Prozent der Engagierten tätig. 8,7 Prozent der Engagierten üben ihre freiwillige Tätigkeit im Rahmen einer staatlichen oder kommunalen Einrichtung aus und 10,2 Prozent in einer anderen formal organisierten Einrichtung. Diesen formal organisierten Varianten freiwilligen Engagements ste-

8 Dieses Unterkapitel wurde verfasst von Corinna Kausmann, DZA; Nadiya Kelle, DZA und Julia Simonson, DZA. 
Abbildung 4.11 Organisationsformen des freiwilligen Engagements, im Zeitvergleich (in Prozent)

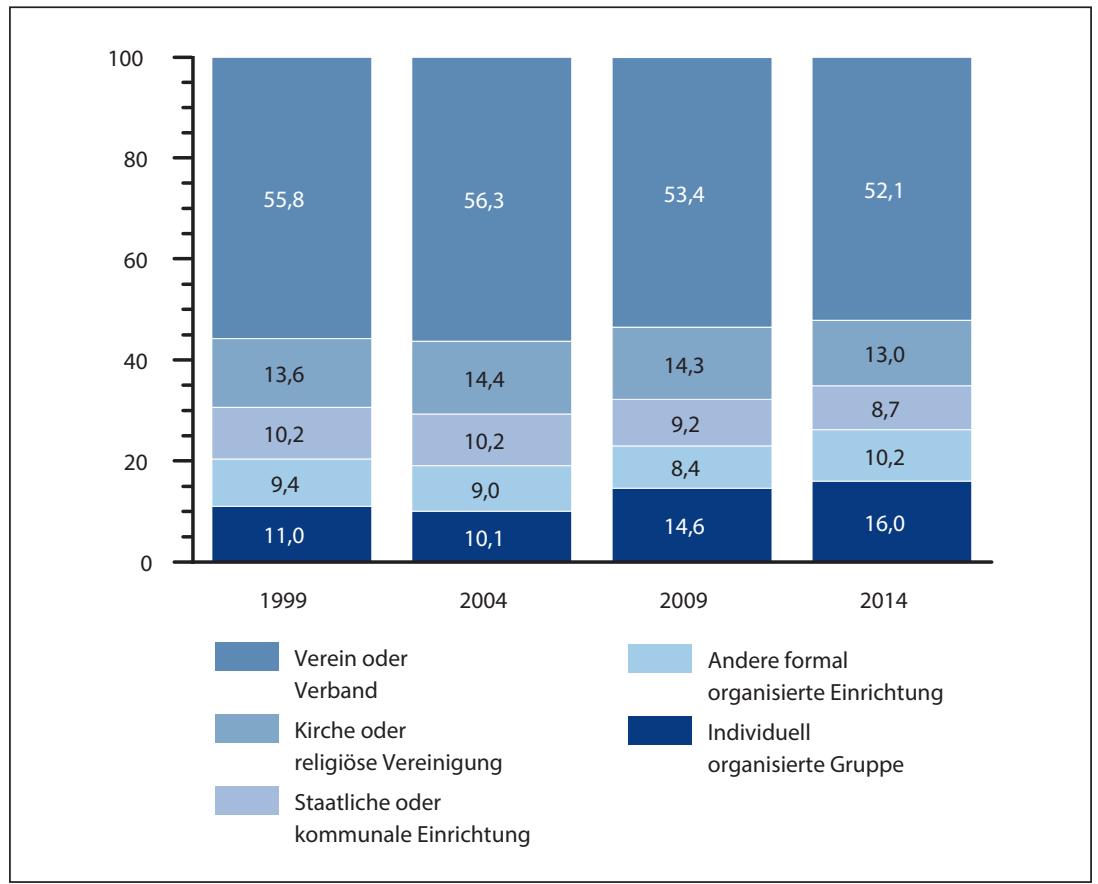

Quelle: FWS, gewichtet, Berechnungen DZA. Basis: Alle Engagierten. FWS 1999 ( $n=4683), 2004$ $(n=5128), 2009(n=6986), 2014(n=12296)$.

hen 16,o Prozent freiwillig Engagierte gegenüber, die sich in individuell organisierten Gruppen engagieren.

Entwicklungen des organisationsgebundenen und informellen Engagements von 1999 bis 2014 (FWS): Im Zeitverlauf sind die Anteile freiwilligen Engagements in formal organisierten Verbänden und Vereinen zurückgegangen: Im Jahr 1999 waren 55,8 Prozent der Engagierten in dieser Form tätig. Im Jahr 2014 sind die Anteile auf 52,1 Prozent gesunken. In den Kirchen oder religiösen Vereinigungen ist die Engagementbeteiligung innerhalb der 15 Jahre wiederum recht stabil geblieben (Abb. 4.11). Die individuell organisierten Gruppen verzeichnen im selben Zeitraum einen Anstieg der Anteile freiwillig Engagierter. Während die Anteile 1999 bei 11,o Prozent lagen, übten 2014 mit 16,o Prozent schon deutlich mehr freiwil- 
lig Engagierte ihre Tätigkeit in diesem Rahmen aus. Die Betrachtung der Verteilung auf die verschiedenen Organisationsformen geschieht auf Basis der freiwillig Engagierten. Berücksichtigt man, dass der Anteil der freiwillig Engagierten im Zeitvergleich insgesamt angestiegen ist, so zeigt sich, bezogen auf die gesamte Wohnbevölkerung ab 14 Jahren in Deutschland in allen Organisationsformen ein Anstieg der Personen, die sich freiwillig engagieren. Die rückläufigen relativen Quoten in Vereinen und Verbänden im Zeitvergleich stehen also nicht für eine Verringerung der absoluten Zahl von Engagierten in dieser Organisationsform. Das freiwillige Engagement in individuell organisierten Gruppen ist lediglich stärker angestiegen als das in Vereinen und Verbänden.

Im Allgemeinen engagieren sich Menschen am häufigsten in formalen zivilgesellschaftlichen Organisationen. Mit Daten des FWS lässt sich seit 1999 belegen, dass das freiwillige Engagement am häufigsten in Vereinen und Verbänden organisiert ist. Diese hohen Anteile beruhen auf der großen Anzahl an Vereinen in Deutschland (Krimmer und Priemer 2013). Ebenso sind die Mitgliedschaften in Vereinen und Verbänden in den letzten Jahren angestiegen (Vogel und Hameister 2017). Das wiederum erhöht auch die Wahrscheinlichkeit, sich in diesem Rahmen freiwillig zu engagieren.

\subsection{Zeitintensives freiwilliges Engagement ${ }^{9}$}

Freiwilliges Engagement gestaltet sich hinsichtlich der Tätigkeitsinhalte, Organisationsformen oder auch der zeitlichen Ausgestaltung unterschiedlich. Neben den Angaben, ob und wie sich Personen in Deutschland engagieren, liefern auch die Auskünfte zum zeitlichen Aufwand Informationen zur Struktur des Engagements in Deutschland und wie es sich über die Zeit entwickelt und verändert. Ebenso kann zwischen Personengruppen mit unterschiedlichen Intensitätsstufen des Engagements unterschieden werden, um zu analysieren, inwiefern sich zwischen den Gruppen Engagierter Unterschiede - sei es hinsichtlich des Alters oder der Erwerbstätigkeit - ausmachen lassen. So können beispielsweise Zeitkonkurrenzen in verschiedenen Phasen des Lebens identifiziert werden.

Zeitaufwand für das Engagement in der Zeitverwendungserhebung (ZVE): Anhand der Daten der Zeitverwendungserhebung (ZVE) 2012/2013 ist es möglich, den Zeitaufwand des Engagements systematisch zu erfassen. Auf dieser Grundlage

9 Dieses Unterkapitel wurde verfasst von Luise Burkhardt, DIW (SOEP) und Boris Rump, DOSB. 
Abbildung 4.12 Durchschnittlicher Zeitaufwand der Engagierten ab 14 Jahren, im Zeitvergleich (in Prozent)

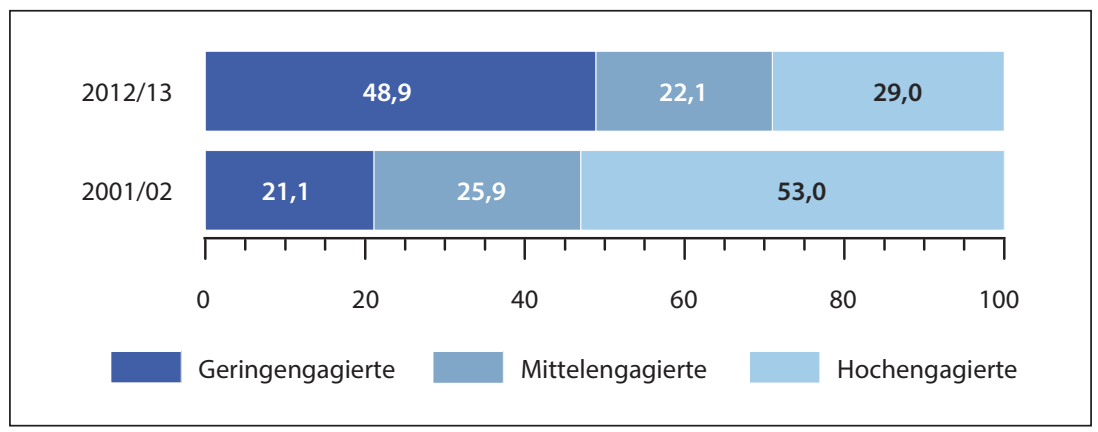

Quelle: ZVE 2012/13, Zeitbudget 2001/02, gewichtet, Berechnungen DIW. Basis: Alle Engagierten ab 14 Jahren in 2012/13 $(n=4316)$, alle Engagierten ab 14 Jahren in 2001/02 $(n=4777)$.

können die Engagierten einer von drei Gruppen zugeordnet werden: Geringengagierte mit einem Aufwand von bis zu fünf Stunden im Monat für das Engagement, Mittelengagierte mit mehr als fünf und bis zu zehn Stunden im Monat und Hochengagierte mit über zehn Stunden im Monat.

In Abbildung 4.12 zeigt sich, dass in den Jahren 2012/2013 von allen engagierten Personen 29, o Prozent Hochengagierte waren. 22,1 Prozent waren im mittleren Maße engagiert und die Geringengagierten machten fast die Hälfte (48,9 Prozent) aller Engagierten aus (vgl. Burkhardt, Priller und Zimmer 2017). Im Vergleich zweier Erhebungswellen zeigt sich, dass das Verhältnis von Hoch- zu Geringengagierten sich über die Zeit nahezu umgekehrt hat. Dieses Ergebnis ist zum Teil sicherlich auf die unterschiedliche Erfassung des Zeitaufwands der Erhebungen von 2001/02 und 2012/13 zurückzuführen. ${ }^{10}$ Gleichzeitig verdeutlicht diese Entwicklung einen Trend, der auch in anderen Erhebungen aufgezeigt wurde. Im Freiwilligensurvey gaben 1999 noch 22,9 Prozent der freiwillig Engagierten sechs und

10 In der Zeitbudgeterhebung von 2001/02 wird neben den Tagebuchaufzeichnungen im Personenfragebogen erfragt: "Sind Sie in einem oder mehreren der nachstehenden Bereiche ehrenamtlich aktiv? Falls ja, geben Sie bitte an, ob Sie sich über die einfache Mitgliedschaft hinaus aktiv beteiligt haben oder ein Amt übernommen haben und wie hoch der durchschnittliche Zeitaufwand in Stunden pro Woche hierfür war." (Gensicke und Geiss 2004, S. 371). Neben der Fragestellung unterscheiden sich ebenfalls die Antwortkategorien bezüglich der Beteiligung und der Zeitaufwendung sowie die gesellschaftlichen Bereiche von der Zeitverwendungserhebung 2012/13 (Gensicke und Geiss 2004; Statistisches Bundesamt 2015). 
mehr Wochenstunden für ihren zeitlichen Aufwand an, dieser Anteil lag 2014 nur noch bei 18,1 Prozent (Hameister et al. 2017).

Zeitaufwand für das Engagement nach Geschlecht, Altersgruppen, Erwerbsstatus und Haushaltskonstellation (ZVE): Die Zeit, die man für Engagement aufwenden kann, hängt auch mit der Lebens- und Erwerbssituation zusammen. In Tabelle 4.1 ist ein Überblick zum Zeitaufwand für das Engagement von Frauen und Männer dargestellt, differenziert nach Altersgruppen, Erwerbsstatus und Haushaltskonstellationen. Männer investieren insgesamt etwas mehr Zeit in ihr Engagement als Frauen. 31,3 Prozent der engagierten Männer wenden zehn Stunden und mehr im Monat für ihr Engagement auf (hochengagiert). Bei den Frauen beträgt dieser

Tabelle 4.1 Zeitaufwand für Engagement (in Prozent)

\begin{tabular}{|c|c|c|c|c|c|c|}
\hline & \multicolumn{3}{|l|}{ Frauen } & \multicolumn{3}{|l|}{ Männer } \\
\hline & $\begin{array}{l}\text { Hoch- } \\
\text { engagierte }\end{array}$ & $\begin{array}{l}\text { Mittel- } \\
\text { engagierte }\end{array}$ & $\begin{array}{l}\text { Gering- } \\
\text { engagierte }\end{array}$ & $\begin{array}{l}\text { Hoch- } \\
\text { engagierte }\end{array}$ & $\begin{array}{l}\text { Mittel- } \\
\text { engagierte }\end{array}$ & $\begin{array}{l}\text { Gering- } \\
\text { engagierte }\end{array}$ \\
\hline $\begin{array}{l}\text { Insgesamt (ab } 14 \\
\text { Jahren) }\end{array}$ & 26,8 & 20,3 & 52,9 & 31,3 & 23,9 & 44,8 \\
\hline \multicolumn{7}{|c|}{ Altersgruppen (ab 14 Jahren) } \\
\hline 14 bis 24 Jahre & 22,8 & 23,0 & 54,2 & 25,9 & 26,3 & 47,9 \\
\hline 25 bis 44 Jahre & 17,3 & 16,3 & 66,4 & 27,4 & 24,7 & 47,9 \\
\hline 45 bis 64 Jahre & 24,6 & 21,1 & 54,3 & 32,4 & 24,2 & 43,4 \\
\hline Ab 65 Jahren & 43,0 & 22,5 & 34,6 & 38,4 & 20,7 & 40,9 \\
\hline \multicolumn{7}{|c|}{ Erwerbsstatus (ab 16 Jahren) } \\
\hline Vollzeit & 19,7 & 19,4 & 60,9 & 27,9 & 26,2 & 45,9 \\
\hline Teilzeit & 16,8 & 21,0 & 62,2 & 28,9 & 22,2 & 48,9 \\
\hline Nicht erwerbstätig & 29,6 & 17,7 & 52,7 & 32,5 & 22,8 & 44,7 \\
\hline Vor-/Ruhestand & 41,8 & 23,3 & 34,9 & 41,2 & 19,0 & 39,8 \\
\hline \multicolumn{7}{|c|}{ Haushaltskonstellation (ab 18 Jahren) } \\
\hline Einpersonen-HH & 32,8 & 24,2 & 43,0 & 31,3 & 27,0 & 41,7 \\
\hline Paare ohne Kinder & 33,8 & 17,8 & 48,5 & 35,8 & 23,5 & 40,8 \\
\hline Alleinerziehende & 16,2 & 25,3 & 58,5 & / & / & / \\
\hline Paare mit mind. 1 Kind & 16,3 & 16,3 & 67,4 & 27,7 & 22,6 & 49,7 \\
\hline
\end{tabular}

Quelle: ZVE 2012/13, gewichtet, Berechnungen DIW. Basis: Alle Engagierten ab 14 Jahren $(\mathrm{n}=4316)$. / nicht dargestellt, da Prozentuierungsbasis $\mathrm{n}<50$. 
Anteil 26,8 Prozent. Mehr als die Hälfte der Frauen (52,9 Prozent) engagiert sich mit einem Zeitumfang von bis zu fünf Stunden im Monat, bei den Männern sind 44,8 Prozent geringengagiert.

Für die Altersgruppen ist zu erkennen, dass bei den engagierten Männern in höheren Altersgruppen der Anteil Hochengagierter größer ist als in den jüngeren Altersgruppen. Auch bei den engagierten Frauen ab 65 Jahren sind die Anteile Hochengagierter größer als bei den jüngeren. Vor allem engagierte Frauen im Alter zwischen 25 und 44 Jahren weisen besonders hohe Anteile an Geringengagierten auf. Erwerbstätige Frauen und Männer sind mit geringerem zeitlichem Umfang engagiert als Nichterwerbstätige oder Personen im Vor- oder Ruhestand. Hierbei sind erwerbstätige Männer häufiger hochengagiert als erwerbstätige Frauen. Bei Frauen und Männern im Vor- und Ruhestand gleichen sich die Anteile der Hochengagierten an und liegen bei 41,8 und 41,2 Prozent.

Je nach Haushaltskonstellation lassen sich deutliche geschlechtsspezifische Unterschiede hinsichtlich der Zeitintensität des Engagements beobachten. Während Frauen und Männer in Einzelpersonen-Haushalten und Paarkonstellationen ohne Kinder zu ähnlichen Anteilen mit über 30 Prozent hochengagiert sind, sind Frauen in Paarkonstellationen mit mindestens einem Kind zu 16,3 und Männer zu 27,7 Prozent hochengagiert. Engagierte Frauen in Paarkonstellationen mit mindestens einem Kind sind deutlich häufiger geringengagiert (67,4 Prozent). Bei den Männern in dieser Haushaltskonstellation liegt dieser Anteil bei 49,7 Prozent.

Zeitaufwand für das Engagement nach verschiedenen Gesellschaftsbereichen (ZVE und SEB): Wie sich der Zeitaufwand der Engagierten in verschiedenen gesellschaftlichen Bereichen darstellt, wird in Abbildung 4.13 wiedergegeben. Im Gesundheitsbereich ist der Anteil der Hochengagierten mit 49,2 Prozent in Relation zu den geringer Engagierten am höchsten. Auch im sozialen und kulturellen Bereich überwiegen die Hochengagierten jene Personen, die mit geringerem zeitlichem Aufwand engagiert sind. Hingegen ist in den Bereichen Kirche und Religion, Freizeit und Geselligkeit sowie Schule und Kindergarten der Anteil der Geringengagierten höher als der Anteil der Hochengagierten. Mit 62,4 Prozent ist der Anteil der Geringengagierten an allen Engagierten im Bereich Schule und Kindergarten am höchsten. Im Bereich Sport und Bewegung sind nicht nur die Anteile der Gering- und Hochengagierten recht ausgeglichen (Abb. 4.13), es ist auch der Bereich, in dem sich anteilig die meisten Menschen freiwillig engagieren (Vogel et al. 2017a). Entsprechend dem Sportentwicklungsbericht (SEB) 2015/2016 engagiert sich im Durchschnitt jeder bzw. jede Ehrenamtliche im Sport 13,4 Stunden pro Monat für seinen bzw. ihren Verein. Bundesweit ergibt sich daraus eine Arbeitsleistung von rund 22,9 Millionen Stunden, die in den Sportvereinen jeden Monat für ehrenamtliche Zwecke erbracht werden (vgl. Breuer und Feiler, 2017). 
Abbildung 4.13 Zeitaufwand für Engagement nach Bereichen, ab 14 Jahren (in Prozent)

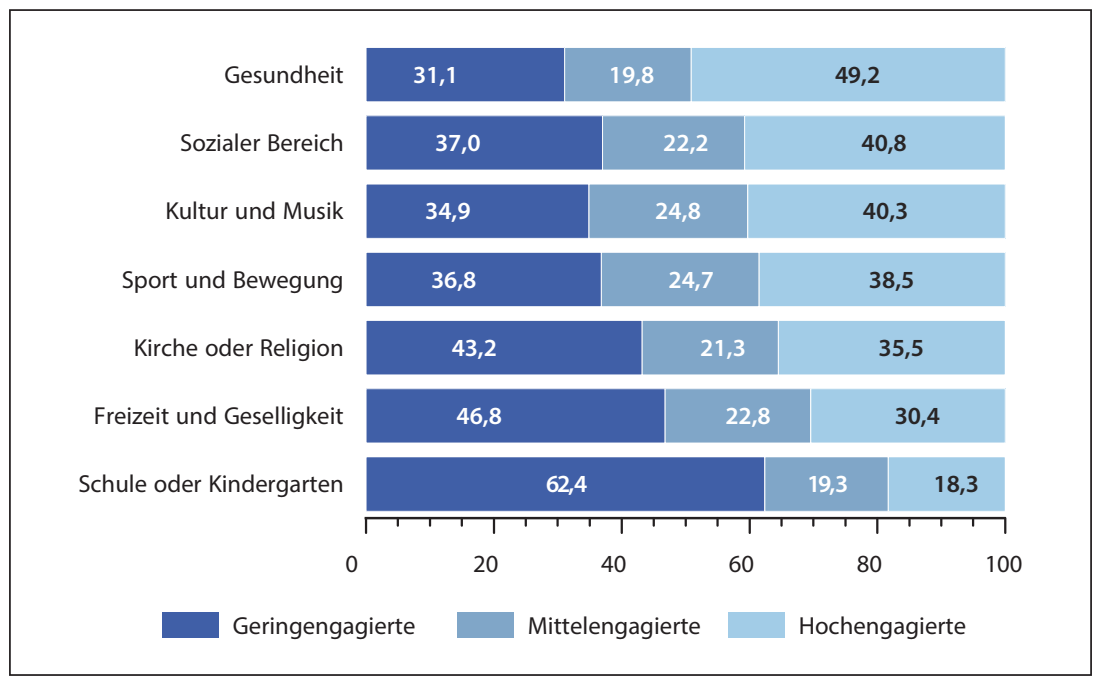

Quelle: ZVE 2012/13, gewichtet, Berechnungen DIW. Basis: Alle Engagierten ab 14 Jahren $(n=4316)$.

Zum einen deuten die Ergebnisse zum Zeitaufwand der Engagierten auf einen möglichen strukturellen Wandel des Engagements hin. Zunehmend mehr Menschen engagieren sich (siehe Abschnitt 4.1), investieren aber in ihre freiwillige Tätigkeit weniger Zeit. Auch andere Studien zeigen, dass das Engagement mehr in zeitlich kürzeren Perioden, eher episodenhaft und projektgebunden und weniger zeitaufwendig sowie weniger an eine kontinuierliche Einbindung in traditionelle Organisationen gekoppelt erfolgt (Ehrhardt 2011; Hameister et al. 2017). Zum anderen zeigt sich, dass der Zeitumfang des Engagements von mehreren Faktoren abhängt. Der Zeitaufwand für das Engagement unterscheidet sich je nach Alter und Lebensphase. Frauen im Alter zwischen 25 und 44 Jahren sind im Altersgruppenvergleich besonders selten hoch- und häufig geringengagiert. Für Männer dieser Altersgruppe ist dieses Phänomen hingegen nicht $\mathrm{zu}$ beobachten. $\mathrm{Zu}$ vermuten ist, dass bei Frauen und Männern unterschiedliche Vereinbarkeitsstrategien bezüglich Karriereplanung, Etablierung auf dem Arbeitsmarkt und Familienarbeit vorliegen, die sich auch auf den Zeitaufwand für Engagement auswirken (Kausmann et al. 2017b). Dieses Ergebnis wird dadurch bekräftigt, dass sich der Anteil an Hochengagierten in der Phase des Vor- und Ruhestandes zwischen den Geschlechtern angleicht. Es ist davon auszugehen, dass durch den Übergang in den Ruhestand und den Ausstieg aus dem Erwerbsmarkt Zeitressourcen frei- 
gesetzt werden, die nun vermehrt in das Engagement investiert werden können. Diese geschlechts- und altersspezifischen Faktoren wirken sich auch auf die unterschiedlichen Gesellschaftsbereiche des Engagements aus. Da bereits die Beteiligung beispielsweise geschlechtsspezifisch geprägt ist (siehe Abschnitt 4.5), können die verschiedenen Lebensphasen den unterschiedlichen Zeitaufwand zusätzlich beeinflussen. Der unterschiedliche Aufwand in den Bereichen kann auch mit den verschiedenen Aufgaben und Tätigkeitsinhalten zusammenhängen, die je nach Bereich, aber auch innerhalb der Bereiche variieren können.

\subsection{Freiwilligendienste ${ }^{11}$}

Freiwilliges Engagement findet auch in der institutionalisierten Form eines Freiwilligendienstes statt. Freiwilligendienste zeichnen sich dadurch aus, dass sie meist auf die Länge eines Jahres begrenzt und währenddessen sehr zeitintensiv in der Ausübung sind. Die Freiwilligendienste werden als Teilzeit- oder Vollzeitbeschäftigung ausgeübt und sind sozialversicherungsrechtlich mit einer Ausbildung gleichgesetzt (Vogel und Simonson 2017). Zu den Freiwilligendiensten zählen das Freiwillige Soziale Jahr (FSJ), das Freiwillige Ökologische Jahr (FÖJ), der Bundesfreiwilligendienst (BFD) ebenso wie lokale Freiwilligendienste oder auch der Europäische Freiwilligendienst (EFD). Freiwilligendienste, wie auch andere Formen freiwilligen Engagements, sind Orte für lebenslanges Lernen. Sie können sich ebenfalls positiv auf die Aufnahme eines künftigen Engagements auswirken.

Freiwilligendienste im Deutschen Freiwilligensurvey (FWS 2014): Im Freiwilligensurvey 2014 werden drei Formen von Freiwilligendiensten dargestellt: der Jugendfreiwilligendienst (dazu zählen FSJ oder auch FÖJ), der Bundesfreiwilligendienst sowie andere Freiwilligendienste. In Abbildung 4.14 sind die Anteile der Personen ab 18 Jahren dargestellt, die in der Vergangenheit einen Freiwilligendienst geleistet haben oder aktuell einen Freiwilligendienst leisten. Dazu werden die drei Formen sowie die Gesamtsumme aller Arten der Freiwilligendienste aufgezeigt. Insgesamt haben 3,7 Prozent im Jahr 2014 angegeben, dass sie einen Freiwilligendienst geleistet haben oder aktuell leisten. Der größte Anteil ist dabei für Jugendfreiwilligendienste zu verzeichnen.

11 Dieses Unterkapitel wurde verfasst von Corinna Kausmann, DZA; Nadiya Kelle, DZA und Julia Simonson, DZA. 
Abbildung 4.14 Anteile von Personen, die einen Freiwilligendienst geleistet haben oder aktuell leisten, 2014 a) gesamt, nach Geschlecht und nach Alter, b) nach Bildung (in Prozent)

a) nach Geschlecht und nach Alter
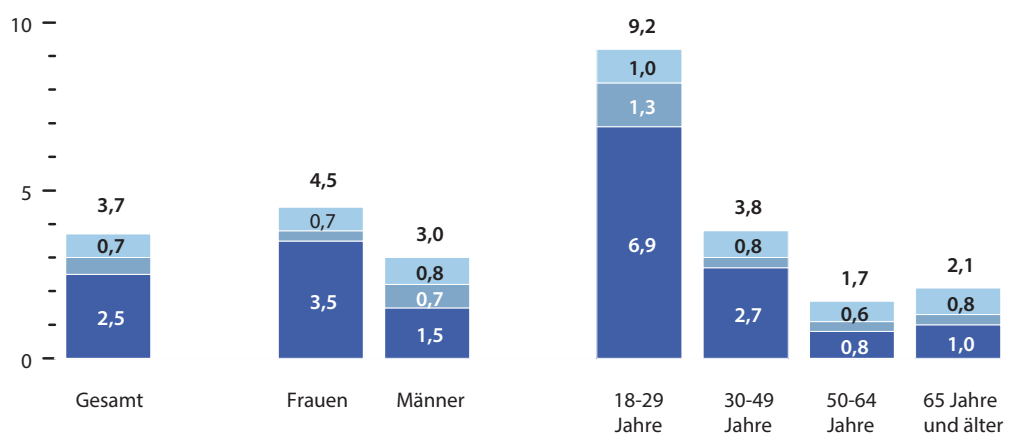

b) nach Bildung
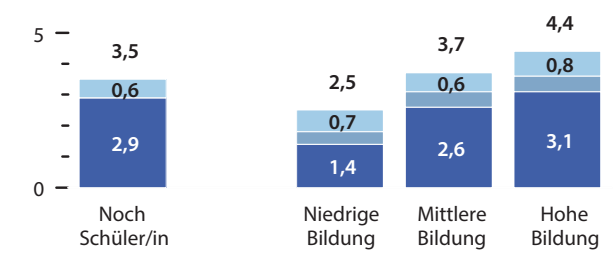

Quelle: FWS 2014, gewichtet, Berechnungen DZA. Basis: Alle Befragten ab 18 Jahre $(n=27314)$.

Freiwilligendienste nach Geschlecht, Alters- und Bildungsgruppen (FWS 2014): Frauen (4,5 Prozent) üben anteilig etwas häufiger einen Freiwilligendienst aus als Männer (3,o Prozent). In den beiden ältesten Altersgruppen (Abb. 4.14a) ist der Anteil der Personen, die sich in einem Freiwilligendienst engagieren oder engagiert haben, historisch bedingt sehr gering (Jugendfreiwilligendienste waren in deren Jugendphase noch nicht etabliert): 1,7 Prozent der Personen im Alter von 50 bis 64 Jahren und 2,1 Prozent der 65 Jahre Alten und Älteren waren in einem Freiwilligendienst tätig. Bei den Personen zwischen 30 bis 49 Jahren liegt der Anteil bei 3,8 Prozent. Am häufigsten sind es die 18- bis 29-Jährigen, die einen Freiwilligendienst ausüben oder ausgeübt haben (9,2 Prozent).

Neben den Altersgruppenunterschieden zeigen sich auch Unterschiede nach schulischer Bildung, die jedoch weniger stark ausgeprägt sind. Wie auch bei an- 
deren Formen von Engagement zeigt sich folgendes Muster: Personen mit hoher Bildung üben häufiger einen Freiwilligendienst aus (4,4 Prozent) als Personen mit mittlerer (3,7 Prozent) oder niedriger Schulbildung (2,5 Prozent; Abb. 4.14b).

Nicht nur für Geschlecht, Alter und Bildung sind Unterschiede in der Beteiligung in Freiwilligendiensten erkennbar, auch die verschiedenen Varianten der Dienste unterscheiden sich zum Teil deutlich voneinander. Der Bundesfreiwilligendienst kann seit seiner Einführung im Jahr 2011 ausgeübt werden und ist somit verständlicherweise noch nicht so stark verbreitet wie die Jugendfreiwilligendienste (Bundesamt für Familie und zivilgesellschaftliche Aufgaben (BAFZA) 2016). Die Jugendfreiwilligendienste haben eine Altersbegrenzung von 27 Jahren. Da es im Bundesfreiwilligendienst keine Altersbegrenzung gibt, kann sich der Anteil der älteren Menschen, die aktuell einen Freiwilligendienst ausüben, in den nächsten Jahren erhöhen. Auch bei den Jüngeren ist ein Anstieg der Beteiligung zu erwarten: Die Wehrpflicht ist ausgesetzt und so könnten mehr junge Männer den Weg in einen Freiwilligendienst finden. Das würde auch zu einer Annäherung in der Beteiligung von Frauen und Männern führen.

\subsection{Literatur}

Alscher, M., \& Priller, E. (2016). Zivilgesellschaftliches Engagement. In Statistisches Bundesamt \& Wissenschaftszentrum Berlin für Sozialforschung (Hrsg.), $D a$ tenreport 2016. Ein Sozialbericht für die Bundesrepublik Deutschland (S. 383389). Bonn: Bundeszentrale für politische Bildung.

Breuer, C., \& Feiler, S. (2017). Sportvereine in Deutschland - Ein Überblick. In C. Breuer (Hrsg.), Sportentwicklungsbericht 2015/2016. Band I. Analyse zur Situation der Sportvereine in Deutschland. (S. 15-46). Hellenthal: Sportverlag Strauß.

Bundesamt für Familie und zivilgesellschaftliche Aufgaben (BAFZA) (2016). Bundesfreiwilligendienst. Online: https://www.bundesfreiwilligendienst.de/der-bun desfreiwilligendienst/oft-gestellte-fragen.html. Zugegriffen: 8. November 2017.

Burkhardt, L., Priller, E., \& Zimmer, A. (2017). Auf der Überholspur? Frauen und freiwilliges Engagement. In Destatis (Hrsg.), Wie die Zeit vergeht. Analysen zur Zeitverwendung in Deutschland (S. 179-193). Wiesbaden: Statistisches Bundesamt (Destatis).

Deutscher Bundestag (2002). Bericht der Enquete-Kommission „Zukunft des Bürgerschaftlichen Engagements". Bürgerschaftliches Engagement: auf dem Weg in eine zukunftsfähige Bürgergesellschaft. (Bundestagsdrucksache 14/8900). Berlin: Deutscher Bundestag.

Deutscher Bundestag (Hrsg.) (2012). Erster Engagementbericht - Für eine Kultur der Mitverantwortung. Bericht der Sachverständigenkommission und Stellungnahme der Bundesregierung (Bundestagsdrucksache 17/10580). Berlin: Deutscher Bundestag. 
Engstler, H., Klaus, D., Lejeune, C., Mahne, K., Spuling, S., Wetzel, M., Wolff, J. K., \& Tesch-Römer, C. (2015). Deutscher Alterssurvey (DEAS): Instrumente der DEASErhebung 2014. Berlin: Deutsches Zentrum für Altersfragen.

Gensicke, T., \& Geiss, S. (2004). Erfassung freiwilligen Engagements (Ehrenamt, Freiwilligenarbeit, Bürgerengagement) in den Freiwilligensurveys 1999/2004 und in der Zeitbudgetstudie. In Statistisches Bundesamt (Hrsg.), Alltag in Deutschland. Analysen zur Zeitverwendung; Beiträge zur Ergebniskonferenz der Zeitbudgeterhebung 2001/o2 am 16./17. Februar 2004 in Wiesbaden. Forum der Bundesstatistik, 43 (S. 357-372). Wiesbaden: Statistisches Bundesamt.

Hagen, C., \& Simonson, J. (2017). Inhaltliche Ausgestaltung und Leitungsfunktionen im freiwilligen Engagement. In J. Simonson, C. Vogel \& C. Tesch-Römer (Hrsg.), Freiwilliges Engagement in Deutschland - Der Deutsche Freiwilligensurvey 2014 (S. 299-331). Wiesbaden: Springer VS.

Hameister, N., Müller, D., \& Ziegelmann, J.P. (2017). Zeitlicher Umfang, Häufigkeit und biographische Dauer des freiwilligen Engagements. In J. Simonson, C. Vogel \& C. Tesch-Römer (Hrsg.), Freiwilliges Engagement in Deutschland - Der Deutsche Freiwilligensurvey 2014 (S. 333-354). Wiesbaden: Springer VS.

International Labour Organization (ILO) (2011). Manual on the Measurement of Volunteer Work. Genf: International Labour Office.

Kausmann, C., Simonson, J., Ziegelmann, J. P., Vogel, C., \& Tesch-Römer, C. (2017a). Länderbericht zum Deutschen Freiwilligensurvey 2014. Wiesbaden: Springer VS.

Kausmann, C., Vogel, C., Hagen, C., \& Simonson, J. (2017b). Freiwilliges Engagement von Frauen und Männern - Genderspezifische Befunde zur Vereinbarkeit von freiwilligem Engagement, Elternschaft und Erwerbstätigkeit. Berlin: Bundesministerium für Familie, Senioren, Frauen und Jugend (BMFSFJ).

Krimmer, H., \& Priemer, J. (2013). ZIVIZ-Survey 2012. Zivilgesellschaft verstehen. Berlin: Stifterverband für die Deutsche Wissenschaft.

Rosenbladt, B. von (2001). Der Freiwilligensurvey 1999. Hinweise und Materialien für Nutzer. Hrsg. vom Projektverbund Ehrenamt und Infratest Burke für GESIS Leibniz-Institut für Sozialwissenschaften. Untersuchung im Auftrag des Bundesministeriums für Familie, Senioren, Frauen und Jugend. München: Infratest Burke.

Roth, R. (2000). Bürgerschaftliches Engagement - Formen, Bedingungen, Perspektiven. In A. Zimmer \& S. Nährlich (Hrsg.), Engagierte Bürgerschaft. Traditionen und Perspektiven (S. 25-48). Opladen: Leske + Budrich.

Simonson, J., \& Hameister, N. (2017). Sozioökonomischer Status und freiwilliges Engagement. In J. Simonson, C. Vogel \& C. Tesch-Römer (Hrsg.), Freiwilliges Engagement in Deutschland - Der Deutsche Freiwilligensurvey 2014 (S. 439-464). Wiesbaden: Springer VS.

Simonson, J., Hameister, N., \& Vogel, C. (2017a). Daten und Methoden des Deutschen Freiwilligensurveys. In J. Simonson, C. Vogel \& C. Tesch-Römer (Hrsg.), Freiwilliges Engagement in Deutschland - Der Deutsche Freiwilligensurvey 2014 (S. 51-88). Wiesbaden: Springer VS. 
Simonson, J., \& Vogel, C. (2017). Organisationale Struktur des freiwilligen Engagements und Verbesserungsmöglichkeiten der Rahmenbedingungen. In J. Simonson, C. Vogel \& C. Tesch-Römer (Hrsg.), Freiwilliges Engagement in Deutschland - Der Deutsche Freiwilligensurvey 2014 (S. 523-548). Wiesbaden: Springer VS.

Simonson, J., Vogel, C., Ziegelmann, J. P., \& Tesch-Römer, C. (2017b). Einleitung: Freiwilliges Engagement in Deutschland. In J. Simonson, C. Vogel \& C. Tesch-Römer (Hrsg.), Freiwilliges Engagement in Deutschland - Der Deutsche Freiwilligensurvey 2014 (S. 31-49). Wiesbaden: Springer VS.

Statistisches Bundesamt (Hrsg.) (2015). Zeitverwendung. Aktivitäten in Stunden und Minuten für ausgewählte Personengruppen. Online: https://www.destatis.de/DE/ Publikationen/Thematisch/EinkommenKonsumLebensbedingungen/Zeitbud geterhebung/Zeitverwendung.html. Zugegriffen: 3. November 2017.

Statistisches Bundesamt (Hrsg.) (2016). Ausgaben und Einnahmen: Länderfinanzausgleich/Bundesergänzungszuweisungen. Online: https://www.destatis.de/DE/Zah lenFakten/GesellschaftStaat/OeffentlicheFinanzenSteuern/OeffentlicheFinan zen/AusgabenEinnahmen/Tabellen/Laenderfinanzausgleich.html. Zugegriffen:

8. November 2017 .

Stricker, M. (2011). Ehrenamt. In T. Olk \& B. Hartnuß (Hrsg.), Handbuch Bürgerschaftliches Engagement (S. 163-171). Weinheim: Beltz Juventa.

TNS Infratest Sozialforschung (2014). SOEP 2013 - Erhebungsinstrumente 2013 (Welle 3o) des Sozio-oekonomischen Panels: Personenfragebogen, Altstichproben. SOEP Survey Papers 180: Series A. Berlin: DIW/SOEP.

UN General Assembly (2001). Recommendations on support for volunteering - Resolution adopted by the General Assembly. A/RES/56/38. New York: UN.

Vogel, C., Hagen, C., Simonson, J., \& Tesch-Römer, C. (2017a). Freiwilliges Engagement und öffentliche gemeinschaftliche Aktivität. In J. Simonson, C. Vogel \& C. Tesch-Römer (Hrsg.), Freiwilliges Engagement in Deutschland - Der Deutsche Freiwilligensurvey 2014 (S. 91-150). Wiesbaden: Springer VS.

Vogel, C., \& Hameister, N. (2017). Mitgliedschaften in Religionsgemeinschaften, in Vereinen und gemeinnützigen Organisationen und freiwilliges Engagement. In J. Simonson, C. Vogel \& C. Tesch-Römer (Hrsg.), Freiwilliges Engagement in Deutschland - Der Deutsche Freiwilligensurvey 2014 (S. 235-251). Wiesbaden: Springer VS.

Vogel, C., Kausmann, C., \& Hagen, C. (2017b). Freiwilliges Engagement älterer Menschen - Sonderauswertungen des Vierten Deutschen Freiwilligensurveys. Berlin: Bundesministerium für Familie, Senioren, Frauen und Jugend (BMFSFJ).

Vogel, C., \& Simonson, J. (2017). Freiwilligendienste als eine Form des freiwilligen Engagements. In J. Simonson, C. Vogel \& C. Tesch-Römer (Hrsg.), Freiwilliges Engagement in Deutschland - Der Deutsche Freiwilligensurvey 2014 (S. 179-197). Wiesbaden: Springer VS.

Vogel, C., Simonson, J., \& Tesch-Römer, C. (2017c). Freiwilliges Engagement und informelle Unterstützungsleistungen von Personen mit Migrationshintergrund. In J. Simonson, C. Vogel \& C. Tesch-Römer (Hrsg.), Freiwilliges Engagement in Deutschland - Der Deutsche Freiwilligensurvey 2014 (S. 253-283). Wiesbaden: Springer VS. 
Vogel, C., Simonson, J., Ziegelmann, J. P., \& Tesch-Römer, C. (2017d). Freiwilliges Engagement von Frauen und Männern in Deutschland. In J. Simonson, C. Vogel \& C. Tesch-Römer (Hrsg.), Freiwilliges Engagement in Deutschland - Der Deutsche Freiwilligensurvey 2014 (S. 637-646). Wiesbaden: Springer VS.

Wetzel, M., \& Simonson, J. (2017). Engagiert bis ins hohe Alter? Organisationsgebundenes ehrenamtliches Engagement in der zweiten Lebenshälfte. In K. Mahne, J. K. Wolff, J. Simonson \& C. Tesch-Römer (Hrsg.), Altern im Wandel: Zwei Jahrzehnte Deutscher Alterssurvey (DEAS) (S. 81-95). Wiesbaden: Springer VS.

Wolff, J.K., Nowossadeck, S., \& Spuling, S.M. (2017). Altern nachfolgende Kohorten gesünder? Selbstberichtete Erkrankungen und funktionale Gesundheit im Kohortenvergleich. In K. Mahne, J. K. Wolff, J. Simonson \& C. Tesch-Römer (Hrsg.), Altern im Wandel: Zwei Jahrzehnte Deutscher Alterssurvey (DEAS) (S. 125-138). Wiesbaden: Springer VS.

Zivilgesellschaft in Zahlen (ZiviZ) (2012). Verteilung der Vereine auf die Bundesländer: ZiviZ Registerdaten 2012, Online: http://www.ziviz.info/fileadmin/download/ ziviz_grafiken_1.pdf. Zugegriffen: 25. April 2018.

Open Access Dieses Kapitel wird unter der Creative Commons Namensnennung 4.0 International Lizenz (http://creativecommons.org/licenses/by/4.0/deed.de) veröffentlicht, welche die Nutzung, Vervielfältigung, Bearbeitung, Verbreitung und Wiedergabe in jeglichem Medium und Format erlaubt, sofern Sie den/die ursprünglichen Autor(en) und die Quelle ordnungsgemäß nennen, einen Link zur Creative Commons Lizenz beifügen und angeben, ob Änderungen vorgenommen wurden.

Die in diesem Kapitel enthaltenen Bilder und sonstiges Drittmaterial unterliegen ebenfalls der genannten Creative Commons Lizenz, sofern sich aus der Abbildungslegende nichts anderes ergibt. Sofern das betreffende Material nicht unter der genannten Creative Commons Lizenz steht und die betreffende Handlung nicht nach gesetzlichen Vorschriften erlaubt ist, ist für die oben aufgeführten Weiterverwendungen des Materials die Einwilligung des jeweiligen Rechteinhabers einzuholen.

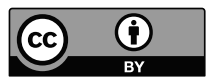

